\title{
Star formation in M 33: the radial and local relations with the gas
}

\author{
S. Verley ${ }^{1,2}$, E. Corbelli ${ }^{1}$, C. Giovanardi ${ }^{1}$, and L. K. Hunt ${ }^{1}$ \\ 1 Osservatorio Astrofisico di Arcetri - INAF, Largo E. Fermi 5, 50125 Firenze, Italy \\ e-mail: [edvige;giova; hunt]@arcetri.astro.it \\ 2 Dept. de Física Teórica y del Cosmos, Facultad de Ciencias, Universidad de Granada, Spain \\ e-mail: simon@ugr.es
}

Received 7 September 2009 / Accepted 29 November 2009

\begin{abstract}
Aims. In the Local Group spiral galaxy M 33, we investigate the correlation between the star formation rate (SFR) surface density, $\Sigma_{\mathrm{SFR}}$, and the gas density $\Sigma_{\text {gas }}$ (molecular, atomic, and total). We also explore whether there are other physical quantities, such as the hydrostatic pressure and dust optical depth, which establish a good correlation with $\Sigma_{\mathrm{SFR}}$.

Methods. We use the $\mathrm{H} \alpha$, far-ultraviolet (FUV), and bolometric emission maps to infer the SFR locally at different spatial scales, and in radial bins using azimuthally averaged values. Most of the local analysis is done using the highest spatial resolution allowed by gas surveys, $180 \mathrm{pc}$. The Kennicutt-Schmidt (KS) law, $\Sigma_{\mathrm{SFR}} \propto \Sigma_{\text {gas }}^{n}$ is analyzed by three statistical methods.

Results. At all spatial scales, with $\mathrm{H} \alpha$ emission as a SFR tracer, the KS indices $n$ are always steeper than those derived with the FUV and bolometric emissions. We attribute this to the lack of $\mathrm{H} \alpha$ emission in low luminosity regions where most stars form in small clusters with an incomplete initial mass function at their high mass end. For azimuthally averaged values the depletion timescale for the molecular gas is constant, and the KS index is $n_{\mathrm{H}_{2}}=1.1 \pm 0.1$. Locally, at a spatial resolution of $180 \mathrm{pc}$, the correlation between $\Sigma_{\mathrm{SFR}}$ and $\Sigma_{\text {gas }}$ is generally poor, even though it is tighter with the molecular and total gas than with the atomic gas alone. Considering only positions where the CO $J=1-0$ line is above the 2- $\sigma$ detection threshold and taking into account uncertainties in $\Sigma_{\mathrm{H}_{2}}$ and $\Sigma_{\mathrm{SFR}}$, we obtain a steeper KS index than obtained with radial averages: $n_{\mathrm{H}_{2}}=2.22 \pm 0.07$ (for FUV and bolometric SFR tracers), flatter than that relative to the total gas $\left(n_{\mathrm{H}_{\mathrm{tot}}}=2.59 \pm 0.05\right)$. The gas depletion timescale is therefore larger in regions of lower $\Sigma_{\mathrm{SFR}}$. Lower KS indices $\left(n_{\mathrm{H}_{2}}=1.46 \pm 0.34\right.$ and $\left.n_{\mathrm{H}_{2}}=1.12\right)$ are found using different fitting techniques, which do not account for individual position uncertainties. At coarser spatial resolutions these indices get slightly steeper, and the correlation improves. We find an almost linear relation and a better correlation coefficient between the local $\Sigma_{\mathrm{SFR}}$ and the ISM hydrostatic pressure or the gas volume density. This suggests that the stellar disk, gravitationally dominant with respect to the gaseous disk in M 33, has a non-marginal role in driving the SFR. However, the tight local correlation that exists between the dust optical depth and the SFR sheds light on the alternative hypothesis that the dust column density is a good tracer of the gas that is prone to star formation.
\end{abstract}

Key words. galaxies: individual: M 33 - galaxies: ISM - Local Group - galaxies: spiral

\section{Introduction}

The gas-to-star conversion process is one of the most important ingredients for galaxy evolution. The rate at which stars form in a galaxy at a given epoch depends not only on the available gas reservoir but also on the ability of the gas to collapse and fragment. The first seminal papers (Schmidt 1959, 1963) related the star formation rate (SFR) to the atomic gas densities using a power law. Comparing the gas density with the number of young stellar objects in the solar neighborhood Schmidt (1959) derived a power law index $n=2$, i.e. $\Sigma_{\mathrm{SFR}} \propto \Sigma_{\text {gas }}^{2}$. Values of $n$ approximately 1.5 to 2.0 were further confirmed by Guibert et al. (1978), using more precise data on the radial and vertical distributions of the interstellar gas and a variety of young stellar objects.

Papers on external galaxies often use the term Schmidt law or Kennicutt-Schmidt (KS) law to relate the surface density of gas to the SFR per unit surface area, since these quantities are the observables for external galaxies. For half a century, numerous studies have been done on the KS law (see Kennicutt 1998a, for a review). For instance, Kennicutt (1989) studied how the globally averaged SFR in a galaxy traced by $\mathrm{H} \alpha$ emission correlates with the mean atomic, molecular, and total $\left(\mathrm{H} \mathrm{I}+\mathrm{H}_{2}\right)$ gas surface densities. For a sample of 15 galaxies they found a good correlation for the atomic and total gas densities
(KS index $n_{\mathrm{H}_{\text {to }}}=1.3 \pm 0.3$ ), but not for the molecular gas density. One decade later, Kennicutt (1998b) studied the relation between the total gas surface density and the $\mathrm{H} \alpha$ SFR density averaged over the entire galaxy, using observations for 61 normal spiral and 36 starburst galaxies. Due to the rather large scatter in the KS relation, the KS index was highly dependent on the method used to fit the data. For the 61 normal galaxies, a leastsquares fit on the SFR density yields $n_{\mathrm{H}_{\mathrm{tot}}}=1.29 \pm 0.18$, while a bivariate least-squares regression leads to $n_{\mathrm{H}_{\mathrm{tot}}}=2.47 \pm 0.39$. Likewise, these two methods to estimate the KS indices for the sample of 36 starburst galaxies lead to values of $n_{\mathrm{H}_{\mathrm{tot}}}=1.28 \pm$ 0.08 and $n_{\mathrm{H}_{\mathrm{tot}}}=1.40 \pm 0.13$, respectively. The better agreement between the two fitting methods mainly reflects the higher gas and SFR dynamic ranges (three orders of magnitude) spanned by the sample of starburst galaxies with respect to the sample of normal galaxies (one order of magnitude). The derivation of the $\mathrm{KS}$ index is very sensitive to the method used to fit the data when there is not a wide dynamical range of the variables.

Today several questions regarding the KS law are still open. First, does the SFR surface density correlate better with the total (atomic plus molecular) gas surface density, as suggested by the globally averaged studies, or only with molecular surface density, since stars condense out of molecular gas? Second, which is the best tracer to characterize the SFR? Is $\mathrm{H} \alpha$ a good local 
current SFR tracer? Does the incompleteness of the IMF at the high mass end in regions where only small clusters form make $\mathrm{H} \alpha$ an unreliable SFR tracer (Corbelli et al. 2009)? The far-UV radiation traces recent SFR averaged over a longer period of time (up to $100 \mathrm{Myr}$ ): how well does it correlate with the gas density locally? On which scale and at which wavelength does the infrared radiation trace star formation? How much of the evolved stellar population is contributing to dust heating?

Third, the dependence of the KS law on the spatial scale considered is not yet clear. It is of interest to study at which scale the KS law might break down and how the power law index $n$ changes as we sample smaller and smaller regions. A first step towards a spatially resolved KS law was performed using azimuthally averaged values of SFR and gas densities (e.g. Martin \& Kennicutt 2001; Wong \& Blitz 2002; Boissier et al. 2003, 2007). For instance, Wong \& Blitz (2002) studied a sample of molecule-rich spiral galaxies and found that the SFR density is more strongly correlated with the $\mathrm{H}_{2}$ surface density than with the total gas surface density. They derived $n_{\mathrm{H}_{2}}=1-2$ with an average value of 1.4 using the molecular gas surface density and a radially varying extinction correction. For the total gas surface density the average KS index found by the same authors is steeper, $n_{\mathrm{H}_{\mathrm{tot}}} \sim 1.7$.

As the resolution of the telescopes increased, the KS law has been examined locally at several spatial resolutions (e.g. Calzetti et al. 2005; Bigiel et al. 2008). However, feedback processes linked to SF activity cast doubt on the applicability of the KS law on very small scales. Photodissociation or photoionization radiation from massive stars or stellar winds can locally remove the molecular hydrogen and make $\mathrm{H}$ II regions not spatially coincident with peaks of CO emission. This effect is visible in M 33 for example when comparing the $\mathrm{H} \alpha$ emission map of this galaxy with CO $J=1-0$ line maps (Engargiola et al. 2003; Heyer et al. 2004). M 33 is a galaxy with a generally low extinction, and the recent Spitzer maps of this galaxy at 8 and $24 \mu \mathrm{m}$ emission confirm that the displacement is not due to embedded H II regions (Verley et al. 2009; Corbelli et al. 2009).

In order to address these questions, recent space mission data in the UV (GALEX, Gil de Paz et al. 2007) and in the IR (Spitzer, Werner et al. 2004) have been used together with ground-based maps in the $\mathrm{H} \alpha$ recombination line. Such a multiwavelength database enables accurate diagnostics of the SFR at high angular resolution in nearby galaxies. The H I Nearby Galaxy Survey (THINGS, Walter et al. 2008) has provided H I and CO maps for many nearby galaxies at an angular resolution of $500-800 \mathrm{pc}$, thus offering the possibility to examine the relation between the gas surface density and SFR density locally, on smaller spatial scales. Bigiel et al. (2008) find that the best correlation is between the $\mathrm{H}_{2}$ surface density and the SFR (traced by a combination of the far-UV and IR surface brightness). The average $\mathrm{KS}$ index is: $1.0 \pm 0.2$. The value $n_{\mathrm{H}_{2}}=1$ implies that the SFR scales with the mass surface density of molecular gas, i.e., that the timescale and the efficiency of SF (the fraction of gas mass converted into stars) is constant.

The THINGS result of unit slope differs from what has been found in other nearby galaxies by other groups using multiwavelength data. Thilker et al. (2007) derive a KS index of 1.64 (1.87) using $\Sigma_{\mathrm{H}_{2}}\left(\Sigma_{\mathrm{H}_{\mathrm{I}}+\mathrm{H}_{2}}\right)$ in molecular dominated regions of NGC 7331. In M 51 a KS index of 1.56 has been found by Kennicutt et al. (2007) for the spatially resolved SFR density on 500 pc scales. The index refers to the total gas density and has been derived by taking into account only bright $\mathrm{H}$ II regions in the center or along the arms of M51. The KS index for the $\mathrm{H}_{2}$ surface density in M 51 is slightly lower, 1.37, very similar to that found for azimuthally-averaged quantities in the BIMA sample or previously in M 33 (Wong \& Blitz 2002; Heyer et al. 2004). In M 31 (Braun et al. 2009) the best correlation at $113 \mathrm{pc}$ scales is established between the SFR density and the total gas density, with a KS index similar to that found for M51. The different power law indices found in the literature, $1 \leq n \leq 3.5$, imply that either the SF law differs from galaxy to galaxy (or from region to region such as arms versus whole disk) or that the KS law index is very sensitive to the method used for deriving it (spatial scale, SFR indicator, extinction corrections, $\mathrm{CO}$ to $\mathrm{H}_{2}$ conversion, background subtraction, etc.).

Due to its proximity, large angular size, and rather low inclination, the Local Group spiral galaxy M 33 is a unique target to investigate the physics underlying the KS law in a latetype galaxy. M 33 has about 1/3 of its baryons in gaseous form, a small molecular fraction and a low dust abundance (Corbelli 2003; Verley et al. 2009). Early attempts to test the KS law in M 33 considered only the atomic gas because of its low molecular content, given the limited spatial resolution and sensitivity of molecular gas observations. In the early 1970's, using stars and $\mathrm{H}$ II region counts together with neutral hydrogen gas, Madore et al. (1974), found a KS index $n_{\mathrm{H}_{\mathrm{I}}} \sim 2.35 \pm 0.26$. Newton (1980) noticed that the density of H II regions on scales of $\sim 300 \mathrm{pc}$ had a weaker dependence on the H I surface density in the inner regions of the galaxy than in the outer ones. More recently, using the first unbiased census of ${ }^{12} \mathrm{CO} J=1-0$ line emission in M 33 and the far-infrared emission map provided by IRAS, Heyer et al. (2004) found a strong correlation between the azimuthally averaged SFR density and the average molecular gas surface computed for annular regions $250 \mathrm{pc}$ wide. For the molecular gas surface density, the KS index is $n_{\mathrm{H}_{2}}=1.36 \pm$ 0.08 , while for the total gas surface density, it is much steeper, $n_{\mathrm{H}_{\mathrm{tot}}}=3.3 \pm 0.07$. This steeper index is consistent with the molecular index, given the low molecular gas fraction in this galaxy which seems regulated by the balance between the gas pressure (acting on the $\mathrm{H}_{2}$ formation rate) and the dissociation radiation (Elmegreen 1993a; Wong \& Blitz 2002; Heyer et al. 2004; Blitz \& Rosolowsky 2006).

Another feature of M 33 that makes it interesting for such a study is the inconsistency of the disk stability with the ongoing SF (Kennicutt 1989; Martin \& Kennicutt 2001). For many years most of the star-forming disk of M 33 has been known to be stable according to the simple Toomre gravitational stability criterion (Toomre 1964) if only the gas surface density is considered (Elmegreen 1993a). Corbelli (2003) has shown however that the Toomre criterion predicts correctly the size of the unstable star forming region of the M 33 disk when the stellar gravity is considered in addition to that of the gaseous disk. Apparently the stellar disk plays an important role in driving the disk instabilities which trigger SF. Its gravity compresses the gas and it can affect the SFR as well. If, for example, the density, or the free fall time, of a cloud depends on the disk gravity perpendicular to the plane, then the SFR density might not correlate with the gas density alone. Also if SF happens only in self gravitating clouds, then the presence of diffuse molecular material in $\mathrm{CO}$ all disk surveys might weaken the expected correlation.

The wealth and quality of newly available data for M 33 has drastically increased during the past years thanks to recent space missions in the UV (GALEX, Gil de Paz et al. 2007) and in the IR (Spitzer, Werner et al. 2004). The high resolution and sensitivity of the recent multiwavelength database for M 33 (Verley et al. 2007, 2009) makes it an ideal target for investigating the radial and local relations between the various SFR tracers and gas densities. The spatial resolution of the CO $J=1-0$ survey 
(Corbelli 2003; Heyer et al. 2004) of M 33 is 45 arcsec, similar to the resolution achieved by Spitzer at $160 \mu \mathrm{m}(\sim 40 \operatorname{arcsec})$. We can therefore test the KS law at a spatial resolution of $180 \mathrm{pc}$. To shed light on the physical basis of the scaling relation between SFR and gas density in late type spiral galaxies, we test whether a simple relation between SFR and gas surface density exists in M 33. We do this using several SFR tracers and spatial resolutions applying different fitting methods. Galaxies such as M 33, which do not have a high SFR per unit surface area and have a nearly constant gas surface density, might not establish a tight KS relation; hence the difference between various fitting methods can be large.

This article is the fourth in a series dedicated to the SF in M 33, after Verley et al. (2007, hereafter Paper I), Verley et al. (2009, hereafter Paper II) and Corbelli et al. (2009, hereafter Paper III). It is organized as follows: Sect. 2 presents the data and the methodology used to derive SFRs and gas surface densities. Section 3 compares the neutral gas distribution, atomic and molecular, with the $\mathrm{H} \alpha$ emission line map. In Sect. 4 we study the KS law considering azimuthal averages of different SFR and gas surface density tracers, and in Sect. 5 we examine the local KS law at various spatial resolutions, using two different methods. In Sect. 6 we investigate whether the SFR per unit area establishes a better correlation with the gas volume density, and we outline possible biases when using $\mathrm{H} \alpha$ emission as SFR tracer on a local scale and the CO line luminosities as molecular gas tracer. Our conclusions are summarized in Sect. 7.

\section{The data sets and methodology}

Here we describe the multiwavelength data set that has been compiled and also the photometric methods and SFR diagnostics. These diagnostics will be used both for the azimuthallyaveraged KS law and for the local KS law on a series of increasing spatial scales.

\subsection{Ultraviolet and $\mathrm{H} \alpha$ line images}

To investigate the continuum ultraviolet (UV) emission of M 33, we use Galaxy Evolution Explorer (GALEX) mission (Martin et al. 2005) data, in particular the data distributed by Gil de Paz et al. (2007). A description of GALEX observations in the far-UV (FUV, 1350-1750 ̊) and near-UV (NUV, 1750-2750 ̊) relative to M 33 and of the data reduction and calibration procedure can be found in Thilker et al. (2005).

To trace ionized gas, we adopt the narrow-line $\mathrm{H} \alpha$ image of M 33 obtained by Greenawalt (1998). The reduction process, using standard IRAF ${ }^{1}$ procedures to subtract the continuum emission, is described in detail in Hoopes \& Walterbos (2000). The total field of view of the image is $1.75 \times 1.75 \mathrm{deg}^{2}(2048 \times$ 2048 pixels of 2 '. 028 on a side).

\subsection{Infrared images}

Dust emission can be investigated through the mid- and FIR data of M 33 obtained with the Multiband Imaging Photometer for Spitzer (MIPS) instrument (Werner et al. 2004; Rieke et al. 2004). The complete set of MIPS $(24,70$, and $160 \mu \mathrm{m}) \mathrm{im}-$ ages of M 33 is described in Paper I: the Mopex software

\footnotetext{
${ }^{1}$ IRAF is distributed by the National Optical Astronomy Observatories, which are operated by the Association of Universities for Research in Astronomy, Inc., under cooperative agreement with the National Science Foundation.
}

(Makovoz \& Marleau 2005) was used to gather and reduce the Basic Calibrated Data (BCD). We chose a common pixel size equal to 1'.2 for all images. The images were background subtracted, as explained in Paper I. The spatial resolutions measured on the images are $6 ", 16^{\prime \prime}$, and $40^{\prime \prime}$ for MIPS 24,70 , and $160 \mu \mathrm{m}$, respectively. The complete field-of-view observed by Spitzer is very large and allows us to achieve high redundancy and a complete picture of the star forming disk of M 33, despite its relatively large extension on the sky.

\subsection{1-cm and millimeter data}

Several data sets are available to examine the atomic and molecular gas distributions: these include the Westerbork Radio Synthesis Telescope (WRST) array data (Deul \& van der Hulst 1987, 24" $\times 48^{\prime \prime}$ spatial resolution) and Arecibo single dish survey (Corbelli \& Schneider 1997, 4' sp. res.). For the molecular gas emission as traced by the $\mathrm{CO} J=1-0$ rotational line, we can use the Berkeley Illinois Maryland Association (BIMA) array data (Engargiola et al. 2003, 13" sp. res.) and the Five College Radio Astronomy Observatory (FCRAO) single dish data (Corbelli 2003; Heyer et al. 2004, 45" sp. res.) or the map obtained by combining the two surveys as described by Rosolowsky et al. (2007). The interferometers in general recover less flux than single-dish data, since they tend to filter the diffuse emission from structures much more extended than the primary beam resolution. In fact, BIMA observations of M 33 contain roughly half of the single-dish flux (Engargiola et al. 2003); this implies that single-dish data are potentially more reliable to measure the total gas column density in M 33, even though it is not clear which one establishes a better correlation with the SFR. We shall use the WRST and the FCRAO data for the atomic and molecular gas distributions respectively, which have a comparable spatial resolution. Following Corbelli (2003), the $\mathrm{CO}$ measurements were converted to $\mathrm{H}_{2}$ mass column densities $\left(M_{\odot} \mathrm{pc}^{-2}\right)$, using the standard conversion factor, $X=$ $2.8 \times 10^{20} \mathrm{~cm}^{-2}\left(\mathrm{~K} \mathrm{~km} \mathrm{~s}^{-1}\right)^{-1}$.

\subsection{Star formation tracers}

We can use several SF tracers to test the KS law. Different tracers are sensitive to different timescales of SF, and their accuracy to trace SF episodes strongly depends on the SF history and dust content of the galaxy under scrutiny. $\mathrm{H} \alpha$ emission traces gas ionized by massive stars in recent bursts of star formation over timescales of $10 \mathrm{Myr}$ or so. The FUV luminosity corresponds to relatively young stellar populations ( $\leq 100 \mathrm{Myr}$ ), so can be considered as complementary to $\mathrm{H} \alpha$ in terms of sensitivity to short timescales.

To convert $\mathrm{H} \alpha$ and FUV emission into SFR, we first correct both $\mathrm{H} \alpha$ and FUV for extinction using the formalism developed by Calzetti (2001). This empirical approach relates the extinction to the TIR and FUV luminosities. The TIR flux (in $\mathrm{W} \mathrm{m}^{-2} \mathrm{pc}^{-2}$ ) is the total IR flux from 3 to $1000 \mu \mathrm{m}$ (Dale \& Helou 2002) defined as:

$F(\mathrm{TIR})=10^{-14} \times\left[19.5 F_{v}(24)+3.3 F_{v}(70)+2.6 F_{v}(160)\right]$,

where $F_{v}(24), F_{v}(70)$, and $F_{v}(160)$ are the MIPS flux densities in $\mathrm{Jy} \mathrm{pc}^{-2}$ (see also Paper II). The extinction correction can then be written as:

$A_{\mathrm{FUV}}=2.5 \times C \times \log \left(\frac{1}{1.68} \times \frac{L(\mathrm{TIR})}{L(\mathrm{FUV})}+1\right)$ 
The value of $C$ is unity if considering star forming regions. Since we sample star forming regions and the ISM with older populations, we adopt an average value $C=0.7$ (Paper II). For $\mathrm{H} \alpha$ extinction we shall use $A_{\mathrm{H} \alpha}=0.3 \times A_{\mathrm{FUV}}$. Following the calibrations given in Paper II, the SFR for the $\mathrm{H} \alpha$ and FUV wavelengths are given by the following formulae:

$$
\begin{aligned}
& \operatorname{SFR}(\mathrm{H} \alpha)\left[M_{\odot} \mathrm{yr}^{-1}\right]=8.3 \times 10^{-42} L(\mathrm{H} \alpha)\left[\mathrm{erg} \mathrm{s}^{-1}\right], \\
& \operatorname{SFR}(\mathrm{FUV})\left[M_{\odot} \mathrm{yr}^{-1}\right]=8.8 \times 10^{-44} L(\mathrm{FUV})\left[\mathrm{erg} \mathrm{s}^{-1}\right]
\end{aligned}
$$

Furthermore, Paper II has shown that in a galaxy of low dust content such as M 33, multifrequency diagnostics can help to determine the SFR more accurately. We adopt one "hybrid" SFR tracer in addition to $\mathrm{H} \alpha$ and FUV tracers: the bolometric luminosity, approximated by a linear combination of the FUV and TIR (Thilker et al. 2007). This is potentially a reliable SF tracer in regions which are more extended than individual H II regions. It reflects both the unobscured and obscured regions of the galaxy (see Paper III) and is sensitive to a variety of starformation timescales. To estimate the bolometric luminosity, we used the following equation, given by Thilker et al. (2007):

$L(\mathrm{bol})=v_{\mathrm{FUV}} L_{v, \mathrm{obs}}(\mathrm{FUV})+(1-\eta) L(\mathrm{TIR})$.

We know that the interstellar radiation field which heats the dust has a contribution from the old stellar population. Some of the IR emission may also come from dust heated by evolved stars (AGB), and thus not be directly associated with recent SF episodes (see Paper II). This heating might dominate for dust outside H II regions. The factor $\eta$ accounts for this (Bell 2003; Iglesias-Páramo et al. 2006), and we shall use $\eta=0.3$. The bolometric SFR was calculated according to Thilker et al. (2007):

$\mathrm{SFR}(\mathrm{bol})\left[M_{\odot} \mathrm{yr}^{-1}\right]=4.6 \times 10^{-44} L(\mathrm{bol})\left[\mathrm{erg} \mathrm{s}^{-1}\right]$.

This equation is based on the calibration of Iglesias-Páramo et al. (2006) derived from Starburst 99 models (Leitherer et al. 1999), with a Salpeter IMF $\left(0.1-100 M_{\odot}\right)$, solar metallicity, and a continuous SF.

\subsection{Radial profiles and aperture photometry}

In Sect. 4 we shall investigate the azimuthally averaged KS law by correlating the azimuthally averaged values of the SFR to gas surface density. Following Heyer et al. (2004), we compute $\Sigma_{\text {SFR }}$ and $\Sigma_{\text {gas }}$ as the mean values within elliptical annuli spaced by $0.24 \mathrm{kpc}$ and centered on the galaxy center $\left(01^{\mathrm{h}} 33^{\mathrm{m}} 50^{\mathrm{s}} .90\right.$, $+30^{\circ} 39^{\prime} 35^{\prime \prime} .8$ ). The annuli are assumed to be circular rings viewed at an inclination of $54^{\circ}$ and with the line of nodes at a position angle of $22.5^{\circ}$, thus representing the spatial orientation of the M 33 disk with respect to the line of sight (McConnachie et al. 2006). We performed this analysis on molecular, atomic and total gas tracers (from FCRAO and WRST data) and for each of the SFR tracers as described in the previous section. These azimuthal averages give $\Sigma_{\mathrm{SFR}}$ and $\Sigma_{\text {gas }}$ as a function of radius, the central radius of each ring. We then correct $\Sigma_{\text {gas }}$ and the radiation emitted from the newly formed stars for the disk inclination to obtain face-on values.

In addition to analyzing azimuthal averages, we shall investigate the local KS law using the highest spatial resolution possible for our dataset (see Sect. 5). This is the spatial resolution of FCRAO CO $J=1-0$ map, similar however to that of 21-cm and $160 \mu \mathrm{m}$ maps. In order to compare $\Sigma_{\mathrm{SFR}}$ and $\Sigma_{\text {gas }}$ locally we first degraded the $24,70,160 \mu \mathrm{m}, \mathrm{FUV}$, and $\mathrm{H} \alpha$ images to the

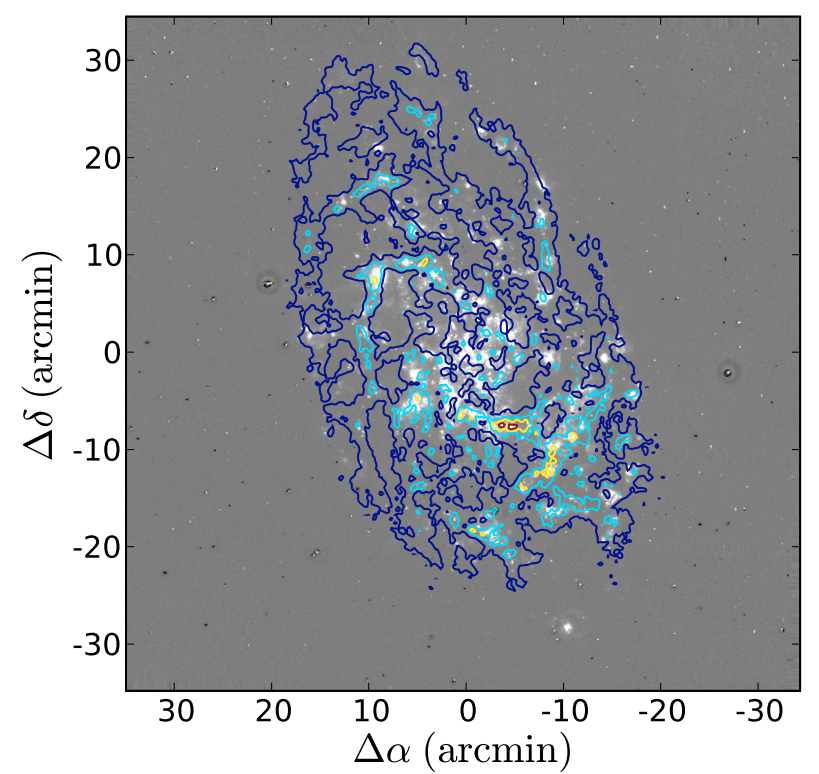

Fig. 1. Equal density H I contours (in logarithm: 0.55, 0.85, 1.03, $1.15 M_{\odot} \mathrm{pc}^{-2}$ ) superimposed over the $\mathrm{H} \alpha$ image.

lowest resolution, i.e. to $45^{\prime \prime}$, corresponding to the spatial resolution of FCRAO molecular map. This corresponds to sizes of $180 \mathrm{pc}$ at the distance of M 33. This is still large enough not to resolve out single molecular clouds: molecular clouds sizes in M 33 are smaller than 100 pc (Rosolowsky et al. 2003). Then, at every position observed in the FCRAO map, we performed aperture photometry on each of the images with a beam of $45^{\prime \prime}$ (full width half maximum). This gives a total of 7664 positions over the disk of M 33. Finally, we converted the photometric results to the appropriate surface density units to examine the local KS law.

To investigate how the KS law varies as a function of the spatial scale we evaluate the KS law on coarser spatial scales by averaging the surface densities over adjacent positions.

\section{Comparison of neutral and ionized gas distributions}

Figure 1 shows the H I contours (at logarithm levels of 0.55 , $0.85,1.03,1.15 M_{\odot} \mathrm{pc}^{-2}$ ) overplotted on the $\mathrm{H} \alpha$ image. The $\mathrm{HI}$ atomic gas distribution in M 33 is very filamentary, and the $\mathrm{H}$ II regions lie on the high surface density H I filaments. But the bright $\mathrm{H} \alpha$ emission knots in M 33 are generally not coincident with the location of the neutral gas peaks. The H I contours follow the $\mathrm{H} \alpha$ emission on large spatial scales, but not locally, on small scales. This agrees with Wright et al. (1972), who found no correlation between the positions of the ten brightest $\mathrm{H}$ II regions and the $\mathrm{H}$ I density peaks. In the northern spiral arm, south of NGC 604, there is relatively strong H I emission, but little $\mathrm{H} \alpha$ emission associated with it, as already noted by Newton (1980). The H I emission extends very far from the center, much beyond the optical disk, with a slower radial decline than that of the $\mathrm{H} \alpha$ surface brightness. The H I contours even trace the northern plume where the $\mathrm{H} \alpha$ emission is faint. The maxima of the H I emission are in the southern half of the galaxy, exactly on the southern arm, and not in the geometrical center of M 33, where the gas mass density is dominated by molecular gas, and several areas appear to be totally devoid of $\mathrm{HI}$. 


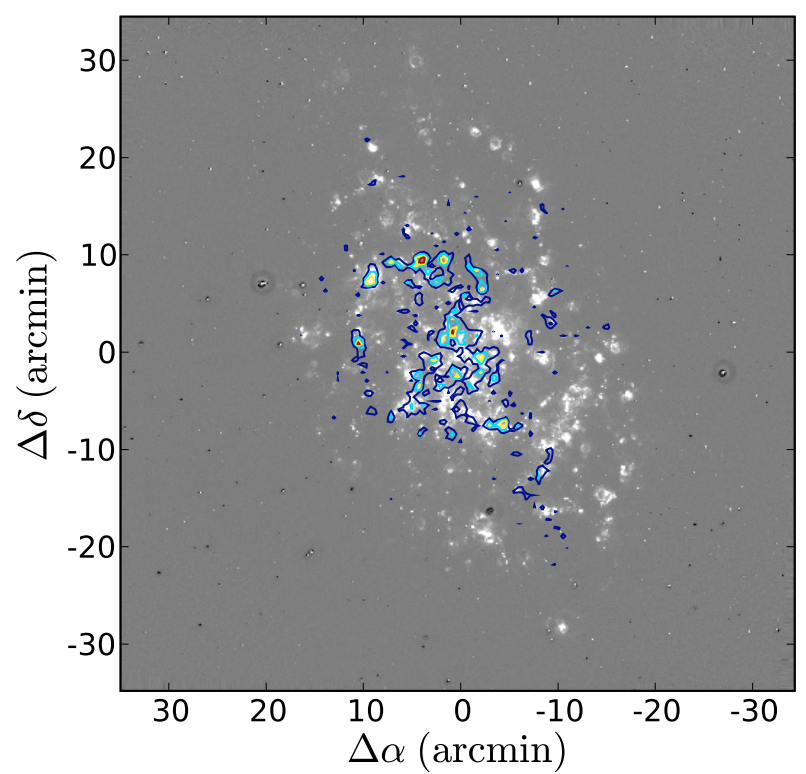

Fig. 2. Equal density CO contours (in logarithm: 0.90, 1.11, 1.25, $1.36 M_{\odot} \mathrm{pc}^{-2}$ ) superimposed over the $\mathrm{H} \alpha$ image.

We use the FCRAO CO $J=1-0$ emission map to trace the $\mathrm{H}_{2}$ molecular gas, with the conversion factor as given in Sect. 2.3. In Fig. 2, the levels of the contours are, in logarithm, $0.90,1.11,1.25,1.36 M_{\odot} \mathrm{pc}^{-2}$. Above the detection threshold of FCRAO measurements $\left(3 \sigma \sim 0.8 \mathrm{~K} \mathrm{~km} \mathrm{~s}^{-1}\right)$, molecular gas is visible only in the inner disk of the galaxy, up to about 3-4 kpc. $\mathrm{CO}$ contours closely follow the southern spiral arm of the galaxy. In the north is a displacement of the $\mathrm{CO}$ emission peaks relative to the arm as traced by $\mathrm{H} \alpha$. This displacement is also evident in the BIMA data of M 33 (Engargiola et al. 2003). For the GMCs of BIMA, Engargiola et al. (2003) showed that there is clustering of GMCs and $\mathrm{H}$ II regions out to a separation of $150 \mathrm{pc}$. But only $67 \%$ of the detected GMCs have their centroid position within $50 \mathrm{pc}$ of an $\mathrm{HII}$ region. Along the minor axis, on the western side of the galaxy, CO is absent, while there are bright $\mathrm{H}$ II regions visible in $\mathrm{H} \alpha$ emission as well as in the IR. No CO peaks are visible close to IC 133, although CO emission is detected around the bright H II region NGC 604.

Unlike luminous spiral galaxies, the gas component of M 33 is dominated by atomic gas (Heyer et al. 2004). The atomicto-molecular fraction is extremely high (1 to 10) compared to molecule-rich galaxies (e.g., 0.05 to 0.4 , Wong \& Blitz 2002). Small molecular clouds have been found beyond $3-4 \mathrm{kpc}$ (Gardan et al. 2007). The paucity of molecular gas at large radii compared to the atomic gas, which has no radial decline, can be explained as the balance between disk hydrostatic pressure and dissociating radiation (Heyer et al. 2004). Smaller clouds form in the outer disk and in some interarm regions, which might have a higher SF efficiency and a shorter dispersion time than elsewhere. GMCs in M 33 comprise less than $30 \%$ of the molecular mass, while in our Galaxy GMCs contain $80 \%$ of the molecular gas, and the fraction of molecular gas mass in GMCs decreases with radius.

\section{Radial Kennicutt-Schmidt law}

We first discuss the radial variations of the KS law by analyzing azimuthally averaged values of the SFR and gas surface density in bins of $0.24 \mathrm{kpc}$. To trace the SFR, we consider each
Table 1. Radially averaged KS indices $(n)$ and Pearson correlation coefficients $(r)$.

\begin{tabular}{ccccccc}
\hline \hline SFR & \multicolumn{2}{c}{ Molecular gas } & \multicolumn{2}{c}{ Atomic gas } & \multicolumn{2}{c}{ Total gas } \\
$n_{\mathrm{H}_{2}}$ & $r_{\mathrm{H}_{2}}$ & $n_{\mathrm{H}_{\mathrm{I}}}$ & $r_{\mathrm{H}_{\mathrm{I}}}$ & & $n_{\mathrm{H}_{\mathrm{tot}}}$ & $r_{\mathrm{H}_{\mathrm{tot}}}$ \\
\hline $\mathrm{H} \alpha$ & $1.3 \pm 0.2$ & 0.87 & $5.6 \pm 0.9$ & 0.76 & $3.6 \pm 0.3$ & 0.92 \\
FUV & $1.1 \pm 0.1$ & 0.94 & $4.1 \pm 0.8$ & 0.73 & $2.9 \pm 0.2$ & 0.95 \\
Bol. & $1.1 \pm 0.1$ & 0.94 & $4.1 \pm 0.8$ & 0.73 & $2.9 \pm 0.2$ & 0.95 \\
\hline mean & $1.2 \pm 0.1$ & 0.92 & $4.6 \pm 0.8$ & 0.74 & $3.1 \pm 0.2$ & 0.94 \\
\hline
\end{tabular}

of the three diagnostics derived in the previous section: extinction corrected $\mathrm{H} \alpha$ (Eq. (3)), extinction corrected FUV (Eq. (4)), and bolometric (Eq. (5)) luminosities. In Table 1 we display the $\mathrm{KS}$ indices for all three SFR diagnostics, with regard to each gas component and to the total gas. The KS indices are rather similar if one considers different SFR tracers; only slightly higher $\mathrm{KS}$ indices are found when the $\mathrm{H} \alpha$ emission is used to trace the SFR. We shall discuss this effect in Sect. 6.

The KS indices (slopes) are steeper for the total gas surface densities (Arecibo plus FCRAO) $\left(n_{\mathrm{H}_{\mathrm{tot}}} \approx 3.1\right)$. Since the atomic gas, the dominant contributor to the total gas surface density, has a shallow radial distribution, we find a very high value of the KS index when we correlate the SFR with the atomic gas surface density alone. A tighter correlation with a shallower slope is found between the azimuthally averaged SFR and the molecular gas surface density with $n_{\mathrm{H}_{2}}=1.2 \pm 0.1$, from the inner disk out to $6 \mathrm{kpc}$. The Robertson \& Kravtsov (2008) simulation results are in remarkable agreement with the radially averaged values of the indices found for the atomic and molecular gas in M 33.

The KS law is graphically presented in Fig. 3, for the molecular, atomic, and total gas as a function of the SFR surface densities calculated from the three diagnostics described above. The gas depletion timescales for a constant SF efficiency are represented by dotted lines. One can see that the depletion timescale for the molecular gas is rather constant and between 0.5 and $1 \mathrm{Gyr}$. On the other hand, the depletion timescales for the atomic and total gas vary widely across the disk of M 33. The fastest depletion timescales (about $0.5 \mathrm{Gyr}$ ) occur near the center of the galaxy where the SF is more pronounced; conversely, the lowest depletion timescales are found in the outer parts of M 33 with values reaching roughly $10 \mathrm{Gyr}$.

Slightly higher indices than what we find using the FUV and bolometric emission have been found by Heyer et al. (2004) by examining radially averaged quantities and a SFR traced by IR emission alone (e.g., $n_{\mathrm{H}_{2}} \approx 1.36$ ). Our KS indices are compatible with those found by Wong \& Blitz (2002) if we consider the molecular gas alone. On the contrary, they are much higher than Wong \& Blitz (2002) for the atomic and total gas, because their sample of galaxies is dominated by molecular gas, while M 33 has less than $10 \%$ of its gas in molecular form.

\section{Local Kennicutt-Schmidt law}

In this section, we first examine the KS law locally at a spatial scale of $180 \mathrm{pc}(45 \operatorname{arcsec})$, which is the resolution of the FCRAO CO $J=1-0$ dataset, and then at larger spatial scales. As in the previous section, we calculate the SFR using the three different tracers and evaluate the correlations with the atomic, molecular, and total gas surface densities. Extinction corrections are applied to the $\mathrm{H} \alpha$ and FUV fluxes at each beam position, and the $\operatorname{SFR}(\mathrm{H} \alpha)$ and $\operatorname{SFR}(\mathrm{FUV})$ are computed as described in Sect. 2.4. At all spatial scales, there is much dispersion in the $\Sigma_{\mathrm{SFR}}-\Sigma_{\text {gas }}$ relations, and the dynamical range of the gas surface 


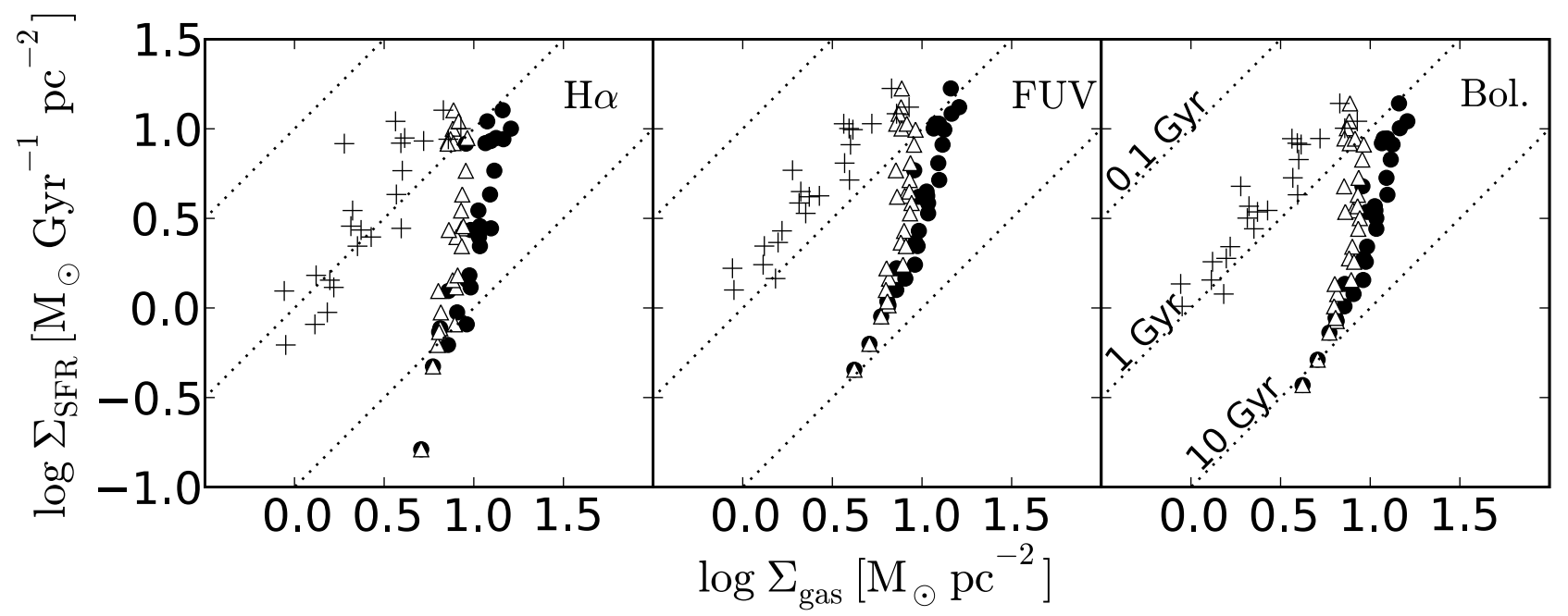

Fig. 3. Radial KS law, elliptically averaged over bins of $0.24 \mathrm{kpc}$. The SFR is calculated from three different tracers: extinction-corrected H $\alpha$ (left), extinction-corrected FUV (center), and bolometric (right). The KS law taking into account only the molecular gas surface density is displayed with plus symbols. The KS law relative to the atomic gas is shown by open triangles. Filled circles corresponds to the KS law when the total gas surface density is considered. The dotted lines represent the gas depletion timescales (cf. labels in Gyr) considering a constant SF efficiency.

density is rather small. As a consequence, the KS indices depend on the fitting method used. We shall give the results from two different fitting methods, which we shall describe in the remainder of this section.

\subsection{Local KS law from 180 to 1440 pc: first fitting method}

To estimate the indices of the KS law at a spatial resolution of $180 \mathrm{pc}$, without taking into account outlier positions with very high or low SFR in our map, we used a recursive fit. The first linear fit includes all the 6400 spatial positions defined by the central square $(80 \times 80$ positions) of the CO map (Heyer et al. 2004). We estimate the dispersion, $\sigma$, of the data with respect to this first fit and remove all points that are lying outside $2 \sigma$ before attempting a second fit. We repeat this action recursively until no points lie outside the $2 \sigma$ dispersion and then quote the slope of this final linear fit. The value of the threshold has been set equal to $2 \sigma$, and changing this threshold could lead to slightly different estimates of the final KS index. Too small a threshold will remove many points at each step and will end up by discarding all the data. On the other hand, if the threshold is too large, the final set of data will remain the same as the initial one, as no points will lie outside the boundaries. Ideally we want to select the value of the threshold which leaves as many points as possible, representing the bulk of the distribution in the final sample while discarding the outliers; a threshold value of $2 \sigma$ seems appropriate for this study (see Figs. 4 and 5) and gives stable results. In order to study the effect of the resolution on the KS indices, we used the same method at coarser spatial resolutions, by averaging the surface densities over adjacent positions: 360 , $540,720,900,1080,1260$, and 1440 pc. In Table 2, we show the final slopes obtained at each resolution for the three SFR tracers, with respect to the molecular, atomic, and total gas. Also in Table 2, we show the Pearson coefficients obtained for the raw samples of points. Clear trends appear: the most significant is that the correlation between $\log \Sigma_{\text {SFR }}$ and $\log \Sigma_{\text {gas }}$ gets better and better when the resolution gets coarser. This was expected due to the stochastic effects which take place at small spatial scales. Although less systematic, the Pearson coefficients are in general higher when considering the molecular gas, with respect to the total and atomic gas. This latter shows the weakest correlation, independently of the SFR tracer used for the correlation.

In Figs. 4 (molecular gas) and 5 (total gas) we display the final slope obtained (heavy line) with the final samples of positions included in the fit, for two cases. Positions discarded (shown in grey) during the several steps of the iteration process lie outside the $2 \sigma$ boundaries (depicted by dashed lines). One can see that the predominant influence on the KS indices is the nature of the gas that is taken into account: the molecular gas gives values of $n_{\mathrm{H}_{2}}$ between 1 and 2, while atomic and total gas exhibit higher values: $n \approx 2-4$. As in the azimuthally averaged relation, the $\mathrm{H} \alpha$ SFR tracer displays higher KS indices than the FUV and bolometric SFR tracers; this will be discussed in the next section.

Indices get steeper on average as the resolution gets more coarse, i.e. as we average quantities over larger areas. For the total gas density the KS indices obtained radially are even higher than the ones obtained for the coarser local resolution. Steeper indices for radially averaged densities are also found by Wong \& Blitz (2002) and Thilker et al. (2007). For the atomic gas the local KS indices are significantly lower, because the H I has a shallow radial falloff, while its distribution in the disk is filamentary. Hence local variations of the atomic gas are more significant than radial variations. This is not the case for the molecular gas, which declines radially with a scalelength of $2 \mathrm{kpc}$, similar to that of the $\mathrm{H} \alpha$ and FUV emission (Paper II). The local KS indices relative to the molecular gas are similar to those found for azimuthal averages in radial bins.

The number of iterations needed to reach the final slope and the data dispersion generally decreases as a coarser spatial resolution is considered (in Table 2 the value of the linear Pearson coefficient $r$ with the original distributions of points, i.e. before the first iteration, is given). This is because stochastic effects weaken the relation between SFR and gas densities at small spatial scales. In the limit of H II region sizes the correlation could disappear altogether, because massive stellar winds and supernovae explosions could remove the molecular gas and quench subsequent SF. Using the combined BIMAFCRAO dataset (Rosolowsky et al. 2007) at a spatial resolution of $30 \operatorname{arcsec}(120 \mathrm{pc})$ around selected $\mathrm{H}$ II regions, we find in fact 
S. Verley et al.: Star formation in M 33: the radial and local relations with the gas

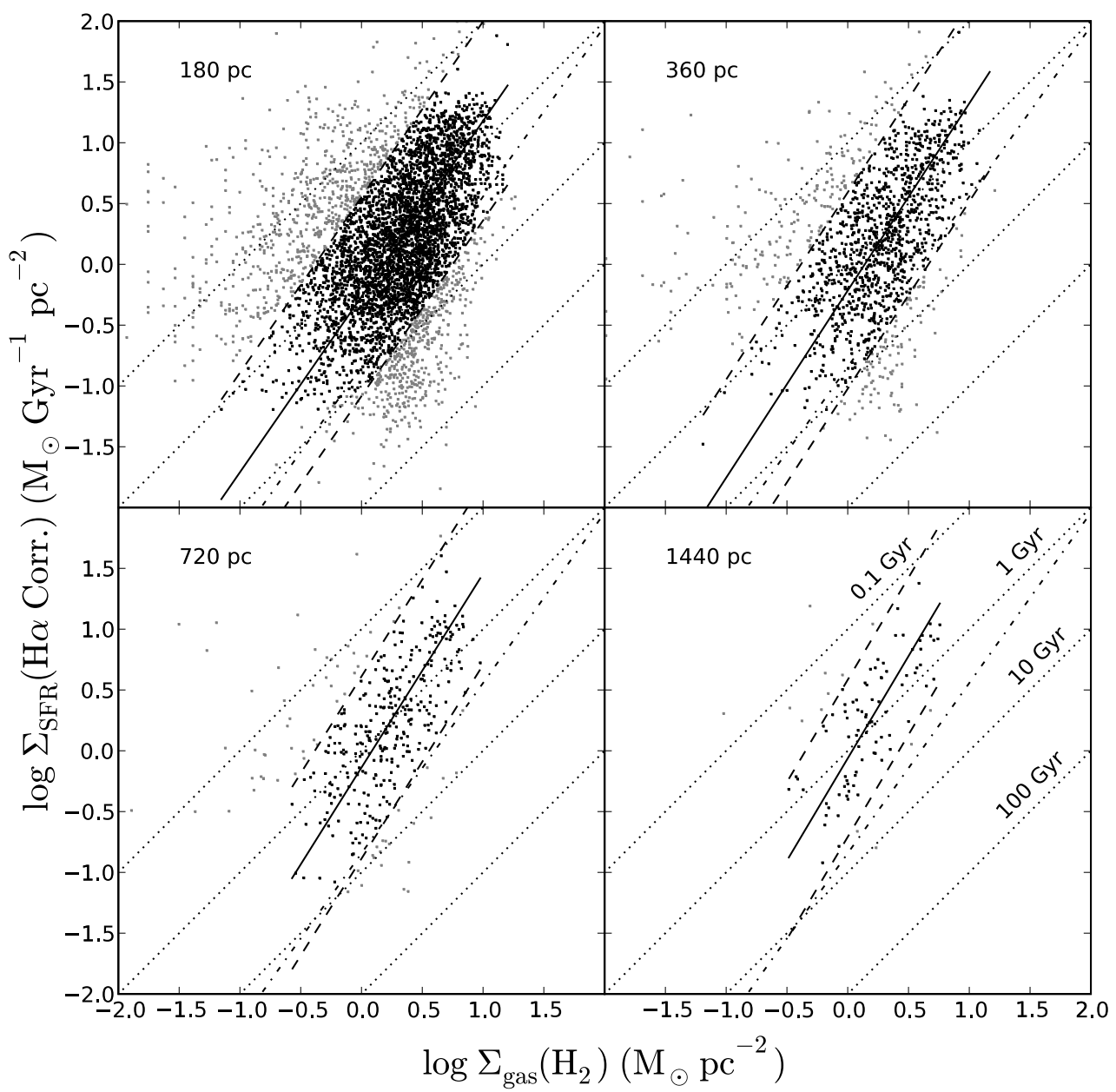

Fig. 4. Local KS law involving the extinction-corrected $\mathrm{H} \alpha$ luminosity as SFR tracer and molecular gas surface density at four spatial resolutions. The black points (inbetween the $2 \sigma$ boundaries, evidenced by the two dashed lines) are the ones considered for the last iteration of the first fitting method. The solid line is the resulting best fit considering these positions. The grey points were discarded during the various iterations of the fit. The Kennicutt (1998b)'s index is depicted by a dash-dot line (slope of 1.4). The dotted lines represent the times of gas depletion (cf. labels in Gyr in the lower right panel) considering a constant SF efficiency.

an even weaker correlation between $\Sigma_{\mathrm{SFR}}$ and $\Sigma_{\mathrm{H}_{2}}$ than over the 180 pc spatial scale.

\subsection{Relative errors: second fitting method}

One caveat of the procedure described in the previous paragraph is that we have considered all positions in the map to allow smoothing and averages over larger areas. This implies that even positions where the molecular gas detection was not above the noise level have been considered. Thus, the reliability of the results on the smallest spatial scale relative to the large-scale ones could be compromised because of low signal-to-noise; as the resolution is degraded, the signal-to-noise ratio increases because we are averaging over larger areas.

We now fit the $\log \Sigma_{\text {SFR }}-\log \Sigma_{\text {gas }}$ local relation at the lowest spatial resolution $(180 \mathrm{pc})$, taking into account the errors in the determination of the gas surface density as well as in the photometry and extinction correction for the determination of the $\Sigma_{\mathrm{SFR}}$. We use the GALEX FUV map to determine the photometric errors ${ }^{2}$. In the smoothed GALEX FUV image the

\footnotetext{
${ }^{2}$ It is not possible to determine the errors for the $\mathrm{H} \alpha$ map since the map we have is in emission measure units and it is not possible to recover the original errors in counts.
}

pixel-to-pixel 1- $\sigma$ noise varies between 2.9 and $8.7 \times$ $10^{-6}$ counts $\mathrm{s}^{-1}$, and we shall consider its maximum value. The maximum large-scale sky $1-\sigma$ variation of sky uncertainty is $3 \times$ $10^{-4}$ counts $\mathrm{s}^{-1}$. The sky noise is much smaller than the largescale sky variation and can be neglected. To the photometric errors we add the errors for extinction corrections. Following Calzetti (2001), we compute the extinction in the GALEX FUV band as in Eq. (2). The largest source of uncertainty in the formula are not the photometric errors on TIR and FUV luminosities, but the bolometric correction factor (1.68). Since the sampled areas contain a mix of young and old stellar populations, it is not clear how much of the TIR luminosity is due to the young stellar population powering the FUV emission. We estimate the uncertainties in $A_{\mathrm{FUV}}$ to be on average $20 \%$. The individual errors on $\mathrm{CO}$ flux estimates can be computed from the rms noise in individual spectra. To these we add the error on the atomic hydrogen surface density, which is on the order of $0.7 M_{\odot} \mathrm{pc}^{-2}$ (as from map noise), if the total gas surface density is considered.

We now consider all areas in M 33 for which the CO flux measured by the FCRAO survey is above $2 \sigma$ uncertainty. We analyze the possible linear correlation between the log of molecular gas surface density and the log of SFR per unit surface as traced by the FUV emission corrected for extinction. A linear 


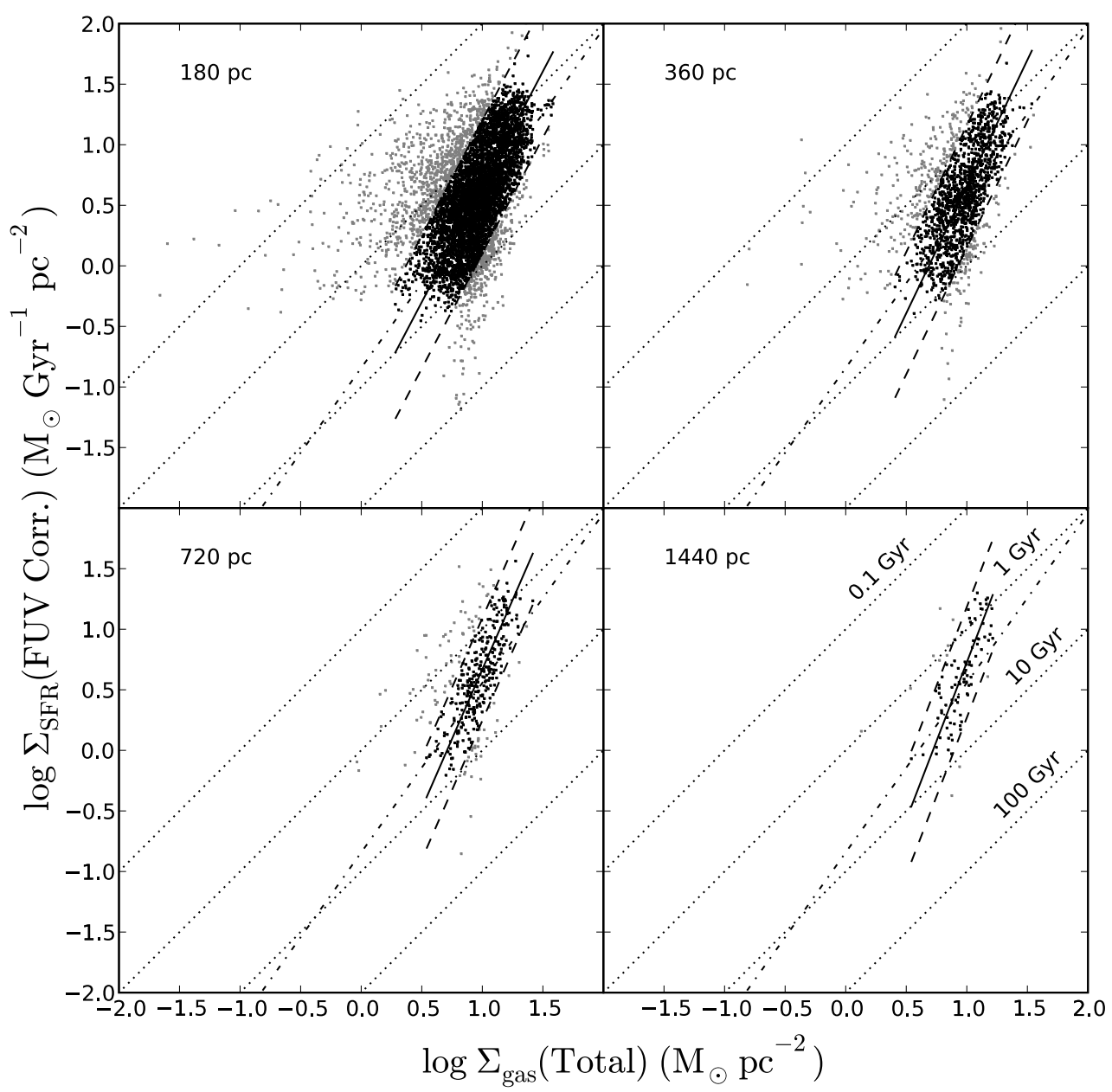

Fig. 5. Local KS law involving the extinction corrected FUV luminosity as SFR tracer and total gas surface density at four spatial resolution. The black points (in between the $2 \sigma$ boundaries, evidenced by the two dashed lines) are the ones considered for the last iteration of the first fitting method. The solid line is the resulting best fit considering these positions. The grey points were discarded during the various iterations of the fit. The Kennicutt (1998b)'s index is depicted by a dash-dot line (slope of 1.4). The dotted lines represent the times of gas depletion (cf. labels in Gyr in the lower right panel) considering a constant SF efficiency.

Table 2. Local KS law indices $(n)$ obtained using the first fitting method for the three SFR tracers.

\begin{tabular}{|c|c|c|c|c|c|c|c|c|c|c|c|c|c|c|c|c|c|c|}
\hline \multirow{3}{*}{$\begin{array}{l}\text { Res. } \\
\text { (pc) }\end{array}$} & \multicolumn{6}{|c|}{ Molecular gas } & \multicolumn{6}{|c|}{ Atomic gas } & \multicolumn{6}{|c|}{ Total gas } \\
\hline & $\mathrm{H} \alpha$ & & FUV & & Bol. & & $\mathrm{H} \alpha$ & & FUV & & Bol. & & $\mathrm{H} \alpha$ & & FUV & & Bol. & \\
\hline & $n$ & $r$ & $n$ & $r$ & $n$ & $r$ & $n$ & $r$ & $n$ & $r$ & $n$ & $r$ & $n$ & $r$ & $n$ & $r$ & $n$ & $r$ \\
\hline 180 & $1.44(43)$ & 0.21 & $1.12(24)$ & 0.24 & $1.12(30)$ & 0.24 & $2.53(14)$ & 0.19 & $1.96(26)$ & 0.23 & $1.96(23)$ & 0.23 & $2.59(18)$ & 0.21 & $1.91(19)$ & 0.26 & $1.90(19)$ & $\overline{0.26}$ \\
\hline 360 & $1.54(16)$ & 0.26 & $1.17(25)$ & 0.29 & $1.17(28)$ & 0.29 & $2.59(12)$ & 0.24 & & 0.29 & & 0.29 & & 0.27 & & 0.32 & & 0.33 \\
\hline 540 & $1.64(20)$ & 0.29 & & 0.33 & & & & 0 . & 2.1 & 0 . & & 0 & & 0.32 & & 38 & & 0.38 \\
\hline 720 & $1.59(7$ & 0.31 & 1) & 0 . & & & 3 & 0.31 & 2.4 & 0 & & 6 & 0) & 0.28 & & 0.33 & & 0.33 \\
\hline 900 & $1.74(10)$ & 0.38 & $1.28(6)$ & 0.40 & 1. & 0 & 10) & 0.32 & $2.09(7)$ & 0.39 & 2. & 0.39 & 3. & 0.44 & $2.22(6)$ & 0.53 & (7) & 0.53 \\
\hline 1080 & $1.81(15)$ & 0.45 & $1.27(13)$ & 0.55 & $1.22(8)$ & 0.55 & $3.62(6)$ & 0.32 & $2.80(7)$ & 0.38 & $2.85(8)$ & 0.38 & 3.98 (13) & 0.48 & $2.27(4)$ & 0.56 & $2.28(4)$ & 0.57 \\
\hline 1260 & $1.62(8)$ & 0.56 & $1.18(4)$ & 0.63 & $1.17(5)$ & 0.64 & $2.77(4)$ & 0.35 & $2.82(5)$ & 0.40 & $2.83(7)$ & 0.40 & $4.08(11)$ & 0.52 & $3.05(11)$ & 0.60 & $2.88(12)$ & 0.60 \\
\hline 1440 & $1.68(3)$ & 0.46 & $1.38(8)$ & 0.51 & $1.39(6)$ & 0.51 & $2.79(2)$ & 0.36 & $2.73(3)$ & 0.46 & $2.74(3)$ & 0.46 & $3.18(3)$ & 0.50 & $2.60(4)$ & 0.61 & $2.61(4)$ & 0.61 \\
\hline
\end{tabular}

Notes. The number of iterations taken before the best-fit slope is stabilized is given in brackets. The Pearson coefficients $(r)$ are the ones obtained with the original distributions of points (i.e. before the first iteration).

least square method with errors both in the gas surface density and in the SFR (subroutine fitexy in Press et al. 1992) gives a slope of $2.22 \pm 0.07$. The Pearson linear correlation coefficient is 0.42 . The error on the slope is determined considering extinction correction error variations: $20 \%$ on average $\pm 5 \%$. The bestfit regression slopes and correlation coefficients relating SFR and gas content in M 33 for the 180 pc regions using this method are given in Table 3. For comparison, the slopes obtained with the ordinary-least-square (OLS) method advocated by Isobe et al. (1990) are also listed in Table 3. This method does not take into account either uncertainties or outliers, so is less sophisticated than either of the two methods described previously. Indeed, the OLS slopes are shallower than either of the other two estimates; in the case of poor correlations with many data points, not 
Table 3. Pearson coefficients $(r)$ and slopes $(n)$ derived from the "relative errors" method for correlations between the two quantities shown in the first column.

\begin{tabular}{lccc}
\hline \hline & $r$ & $n$ & $n_{1}$ \\
\hline $\log \Sigma_{\mathrm{SFR}}-\log \Sigma_{\mathrm{H}_{2}}$ & 0.42 & $2.22 \pm 0.07$ & $1.46 \pm 0.34$ \\
$\log \Sigma_{\mathrm{SFR}}-\log \Sigma_{\mathrm{H}_{\mathrm{I}}}$ & 0.24 & $1.62 \pm 0.03$ & $1.11 \pm 0.15$ \\
$\log \Sigma_{\mathrm{SFR}}-\log \Sigma_{\mathrm{H}_{\text {tot }}}$ & 0.43 & $2.59 \pm 0.05$ & $1.67 \pm 0.25$ \\
$\log \Sigma_{\mathrm{SFR}}-\log \left(\Sigma_{\mathrm{H}_{2}} / \rho_{\mathrm{ISM}}^{-0.5}\right)$ & 0.62 & $1.16 \pm 0.04$ & $1.00 \pm 0.01$ \\
$\log \Sigma_{\mathrm{SFR}}-\log \rho_{\mathrm{ISM}}$ & 0.71 & $1.07 \pm 0.02$ & $0.97 \pm 0.01$ \\
$\log \Sigma_{\mathrm{SFR}}-\log \tau_{160}$ & 0.81 & $1.13 \pm 0.02$ & $1.01 \pm 0.02$ \\
\hline
\end{tabular}

Notes. The SFR per unit area, $\Sigma_{\mathrm{SFR}}$, is traced by the FUV emission corrected for extinction. Only positions where the $\mathrm{CO}$ luminosity is above the $2 \sigma$ limit have been considered at a spatial resolution of $180 \mathrm{pc}$. The surface density of molecular, atomic and total gas are $\Sigma_{\mathrm{H}_{2}}, \Sigma_{\mathrm{H}_{\mathrm{I}}}$ and $\Sigma_{\mathrm{H}_{\mathrm{tot}}}$ respectively. $\rho$ is the volume density of the ISM and $\tau_{160}$ the dust opacity. $n_{1}$ is the OLS slope as described in the text.

considering properly errors or outliers can give too much relative weight to statistically insignificant data. In Fig. 6 we show the $\log \Sigma_{\mathrm{H}_{\mathrm{tot}}}-\log \Sigma_{\mathrm{SFR}}$ relation with the relative best fitting linear relation $(\mathrm{KS}$ index $=2.59 \pm 0.05)$. The correlation with total gas is of similar quality to that with the molecular gas alone, since both Pearson linear correlation coefficients are $\sim 0.43$.

We now consider the correlation between the total gas surface density and the SFR per unit surface, including points which have the $\mathrm{CO}$ brightness below the detection threshold. For these we shall consider only the H I surface density. The correlation coefficient does not change, but the regression is shallower with a slope of $1.7 \pm 0.2$. But caution is called for in interpreting such a shallow slope as real or claiming that there are areas in the galaxy where the surface density decreases but the SFR does not (bi-modal distribution). The non-detection of molecular emission generates artificial tails with zero slopes at low surface densities and flattens the average slope. It is more feasible to consider positions without a reliable $\mathrm{CO}$ detection only when the corresponding molecular surface density threshold is well below the H I surface density, but there are not many positions in our data which satisfy this condition.

\section{Different tracers of the SFR and of the likelihood of the gas to form stars}

In this last section we analyze whether the SFR correlates better with other physical quantities of the ISM, such as the hydrostatic pressure or gas volume density, rather than with the total gas surface density. We also discuss the uncertainties related to the use of the $\mathrm{H} \alpha$ as SFR tracer, which at first seems more appropriate than the FUV or bolometric surface brightness in tracing the most recent star formation. And in the last paragraph we show the tight correlation between the SFR and the dust optical depth. We briefly discuss whether the dust optical depth can trace the gas prone to form stars in the M 33 disk better than the CO $J=1-0$ line intensity.

\subsection{The role of the stellar disk and the dependence of the SFR on the gas volume density}

The widely used KS law relates the SFR per unit surface with the gas surface density. The SFR depends upon the amount of molecular gas able to collapse, fragment and form stars on a timescale $t_{\mathrm{SF}}$. Often the relevant timescale has been referred to

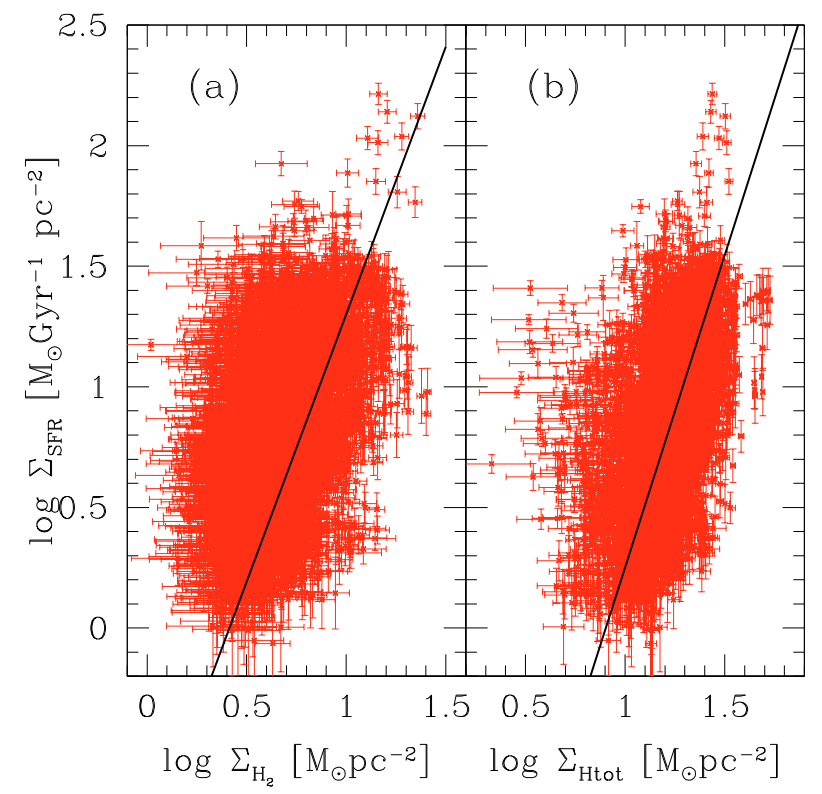

Fig. 6. Local KS law involving the extinction corrected FUV luminosity as SFR tracer and the molecular gas surface density in a), the total gas surface density in b). The spatial resolution is $180 \mathrm{pc}$. The best fitting linear relation leads to a KS index of $2.22 \pm 0.07$ in a) and of $2.59 \pm$ 0.05 in b) shown by the heavy lines.

as the cloud collapse or free-fall timescale, which is inversely proportional to the square root of the gas volume density in the cloud: $t_{\mathrm{SF}} \propto t_{\mathrm{ff}} \propto \rho_{\mathrm{H}_{2}}^{-0.5}$. If $\rho_{\mathrm{H}_{2}}$ scales with some power of the gas surface density or is constant, then it is conceivable that the SFR correlates with the surface density of the self-gravitating gas, $\Sigma_{\mathrm{H}_{2}}$, to some power. However, since the free-fall time is much shorter than the molecular cloud formation timescale (e.g., Ward-Thompson et al. 1994; McKee \& Tan 2003; Engargiola et al. 2003; Krumholz et al. 2009), the relevant time for star formation could be linked to the interstellar medium out of which the clouds form. For example Koyama \& Ostriker (2009) have recently shown using numerical simulations of disks that the ISM structure plays a primary role in determining the actual SFR in galactic disks. To test this hypothesis in M 33, we relate the star formation timescale not to the molecular cloud free-fall time, but to their formation timescale. If $t_{\mathrm{SF}}$ is the time needed to form self-gravitating molecular clouds out of the diffuse interstellar gas, $t_{\mathrm{SF}}$ will depend on the density of the ISM $\rho_{\mathrm{ISM}}$, i.e. $t_{\mathrm{sf}} \propto \rho_{\mathrm{ISM}}^{-0.5}$ (Koyama \& Ostriker 2009). The value of $\rho_{\mathrm{ISM}}$ in the disk scales with the hydrostatic pressure, which in galaxies with a low gaseous content such as M 33 depends on the stellar disk surface density (Corbelli 2003). An important finding in M 33 is that both the outer SF threshold radius and the abundance of molecules can be explained if one includes the contribution of the stellar disk in the hydrostatic pressure equation (Elmegreen 1993b; Corbelli 2003).

In an attempt to improve the local correlation between the gas and the SFR in M 33, we consider the gravitational compression of the gas due to the stellar disk. If the hydrostatic pressure $P$ sets the gas vertical scale height $h_{\mathrm{g}}$ of the disk, the volume density $\rho_{\text {ISM }}$ can be evaluated as $P / c_{\mathrm{g}}^{2}$ or following Corbelli (2003) as:

$\rho_{\mathrm{ISM}}=\frac{\Sigma_{\mathrm{H}_{\mathrm{tot}}}}{2 h_{\mathrm{g}}}$ 
where

$h_{\mathrm{g}}=\frac{c_{\mathrm{g}}}{\pi G}\left(\frac{\Sigma_{\mathrm{H}_{\mathrm{tot}}}}{c_{\mathrm{g}}}+\frac{\Sigma_{\mathrm{s}}}{c_{\mathrm{s}}}\right)^{-1}$

and $\Sigma_{\mathrm{s}}, c_{\mathrm{s}}$ and $c_{\mathrm{g}}$ are respectively the stellar surface density, the velocity dispersion of the stars and the gas perpendicular to the disk. If $\rho_{\text {ISM }}$ determines the SF timescale through the cloud formation timescale (or through $t_{\mathrm{ff}}$ if $\rho_{\mathrm{H}_{2}}$ scales with $\rho_{\mathrm{ISM}}$ ), we expect the following relation to hold:

$\Sigma_{\mathrm{SFR}} \propto \frac{\Sigma_{\mathrm{H}_{2}}}{t_{\mathrm{SF}}} \propto \frac{\Sigma_{\mathrm{H}_{2}}}{\rho_{\mathrm{ISM}}^{-0.5}}$.

We shall consider a gas velocity dispersion of $6 \mathrm{~km} \mathrm{~s}^{-1}$ and a ratio of gas-to-stellar velocity dispersion equal to 0.3 (Corbelli $\&$ Walterbos 2007). By looking at the correlation between $\Sigma_{\mathrm{SFR}}$ and $\Sigma_{\mathrm{H}_{2}} / \rho_{\text {ISM }}^{-0.5}$ we find that this is tighter than that between $\Sigma_{\mathrm{SFR}}$ and the gas surface density alone. The Pearson linear correlation coefficient is 0.62 , and the slope of the linear fit is $1.16 \pm 0.04$ in the $\log -\log$ plane (see Fig. 7), close to linear as expected by the above equation. It seems clear that the gravity of the stellar disk, dominant in M 33, plays a major role in regulating the SFR. Variations of the gas surface density alone are insufficient to explain the scatter in $\Sigma_{\mathrm{SFR}}$ and imply a steep KS index as well as a loose correlation when considering only the gas surface density.

Finally, it is noteworthy that the Pearson linear correlation coefficient is even higher, $r=0.71$, when considering the $\log \Sigma_{\text {SFR }}-\log \rho_{\text {ISM }}$ relation. The slope of the linear correlation, shown in Fig. 7 , is $1.07 \pm 0.02$. The tighter relation between $\Sigma_{\text {SFR }}$ and $\rho_{\text {ISM }}$ (or the hydrostatic pressure since $\rho_{\text {ISM }} \propto P$ ) is well known. It can be interpreted as follows: suppose that $t_{\mathrm{SF}}$ is proportional to $\rho_{\mathrm{ISM}}^{-0.5}$ because the cloud formation timescale is proportional to $\rho_{\mathrm{ISM}}^{-0.5}$ and regulates the SF. If the molecular gas surface density (or volume density if SF is confined into a layer of constant scale height), which is in the form of bound, selfgravitating units, is proportional to $\rho_{\mathrm{ISM}}^{0.57}$, the expected relation is that we find $\Sigma_{\mathrm{SFR}} \propto \rho_{\mathrm{ISM}}^{1.07}$. This would be the case for a constant star formation efficiency.

The surface density of molecular gas in the form of bound, self-gravitating units can be different from $\Sigma_{\mathrm{H}_{2}}$ since $\Sigma_{\mathrm{H}_{2}}$ includes the non negligible contribution of diffuse molecular gas. Unfortunately there are no detailed surveys of the molecular gas in M 33 available as yet which would be able to constrain the fraction of gas in the form of bound, self-gravitating units. Only results of GMCs surveys are available, but the molecular mass spectrum in M 33 is steeper than -2 and hence dominated by molecular clouds of small mass (Blitz \& Rosolowsky 2005).

\subsection{Is Ho luminosity a good SFR tracer on a local scale?}

We compare the three different SFR tracers in Fig. 8, which shows $\Sigma_{\mathrm{SFR}}(\mathrm{H} \alpha)$ plotted against $\Sigma_{\mathrm{SFR}}$ derived from the FUV (left panel) and bolometric luminosity (right) (see Sect. 2). At high SFR densities, $\mathrm{H} \alpha$ and FUV give quite similar values, but $\mathrm{H} \alpha$ underpredicts SFR relative to FUV at low SFR. SFR as inferred from bolometric luminosity is almost always larger than that from $\mathrm{H} \alpha$, and at low SFR densities the effect is quite strong.

With the $\mathrm{H} \alpha$ emission as SFR indicator, the correlation of $\Sigma_{\text {SFR }}$ with $\Sigma_{\text {gas }}$ or $\rho_{\text {ISM }}$ becomes looser, and steeper indices are found. This is to be expected when one samples regions, as we do, with a total bolometric luminosity below $10^{39} \mathrm{erg} \mathrm{s}^{-1}$, and we explain why. The bolometric luminosity of a single cluster or of an ensemble of clusters measures the mass of the clusters

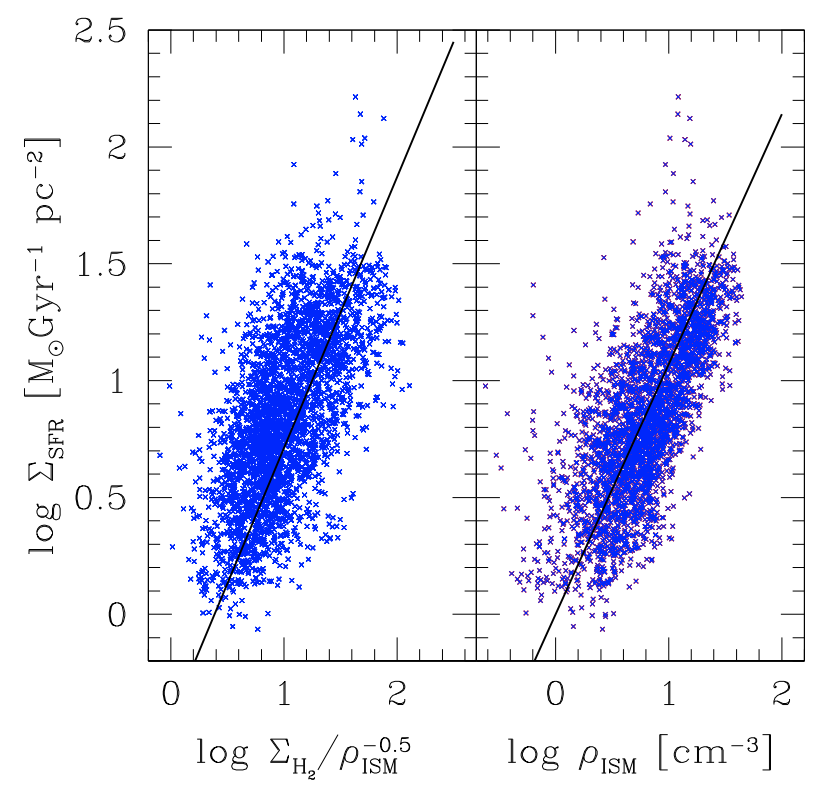

Fig. 7. The left-hand panel shows the linear correlation between $\log \Sigma_{\mathrm{SFR}}$ and $\log \Sigma_{\mathrm{H}_{2}} / \rho_{\mathrm{ISM}}^{-0.5}$ (in units of $M_{\odot} / \mathrm{pc}^{-2} \mathrm{~cm}^{-1.5}$ ). The Pearson linear correlation coefficient is 0.62 , and the slope of the linear fit is $1.16 \pm 0.04$. The slope is derived taking into account uncertainties on both axes, but they are not shown in the graph for clarity. On the right-hand panel we show the linear correlation between $\log \Sigma_{\mathrm{SFR}}$ and $\log \rho_{\text {ISM }}$ with the Pearson coefficient 0.71 .

only when the IMF is fully populated. Hence, when $L_{\text {bol }}$ is comparable to or lower than the luminosity of the most massive star in the cluster, the IMF cannot be fully sampled up to its high mass end.

As Corbelli et al. (2009) pointed out, there are two types of models for populating the stellar clusters when the IMF is incompletely sampled. Either the IMF is truncated to a limiting mass which depends on the cluster mass, or the IMF is incomplete and stochastically sampled, but maintains its original shape and completeness up to its high mass limit over the whole galaxy. For both cases the $L_{\mathrm{bol}} / L_{\mathrm{H} \alpha}$ ratio rises when the most massive stars are lacking because the recombination-line luminosity, $L_{\mathrm{H} \alpha}$ has a steeper dependence on the stellar mass than the bolometric luminosity does. For the first model, the truncation model, a deviation from a simple scaling law between $\log L_{\mathrm{bol}}$ and $\log L_{\mathrm{H} \alpha}$ is expected when $L_{\mathrm{bol}}$ is on the order of the luminosity of the most massive star. For $120 M_{\odot}$ this is about $7 \times 10^{39} \mathrm{erg} \mathrm{s}^{-1}$. For the second model, the randomly sampled cluster model, the stochastic character of the IMF implies that low luminosity clusters can be made either by populating the IMF up to a certain stellar mass, or by just one single star, or by something in between. The presence of outliers makes the average $L_{\mathrm{bol}} / L_{\mathrm{H} \alpha}$ deviate less from its constant value at high luminosities (where the IMF is fully populated). In practice it is only for $L_{\mathrm{bol}} \leq 1.5 \times 10^{39} \mathrm{erg} \mathrm{s}^{-1}$ that $L_{\mathrm{bol}} / L_{\mathrm{H} \alpha}$ drops dramatically. In this regime $L_{\mathrm{H} \alpha}$ is no longer an adequate measure of the cluster mass, and for this model a larger scatter is expected in the bolometric luminosity - cluster mass relation.

Figure 9 shows the theoretical $L_{\mathrm{bol}}-L_{\mathrm{H} \alpha}$ relation, referred to as the cluster birthline. The filled square symbols correspond to the randomly sampled cluster model, and filled circles indicate the prediction for clusters modelled with a truncated IMF (see above and Paper III). Cross symbols in Fig. 9 show the data for the $180 \mathrm{pc}$ regions in $\mathrm{M} 33$. The luminosities of these regions are clearly sufficiently low to enter the regime of an incomplete 
S. Verley et al.: Star formation in M 33: the radial and local relations with the gas

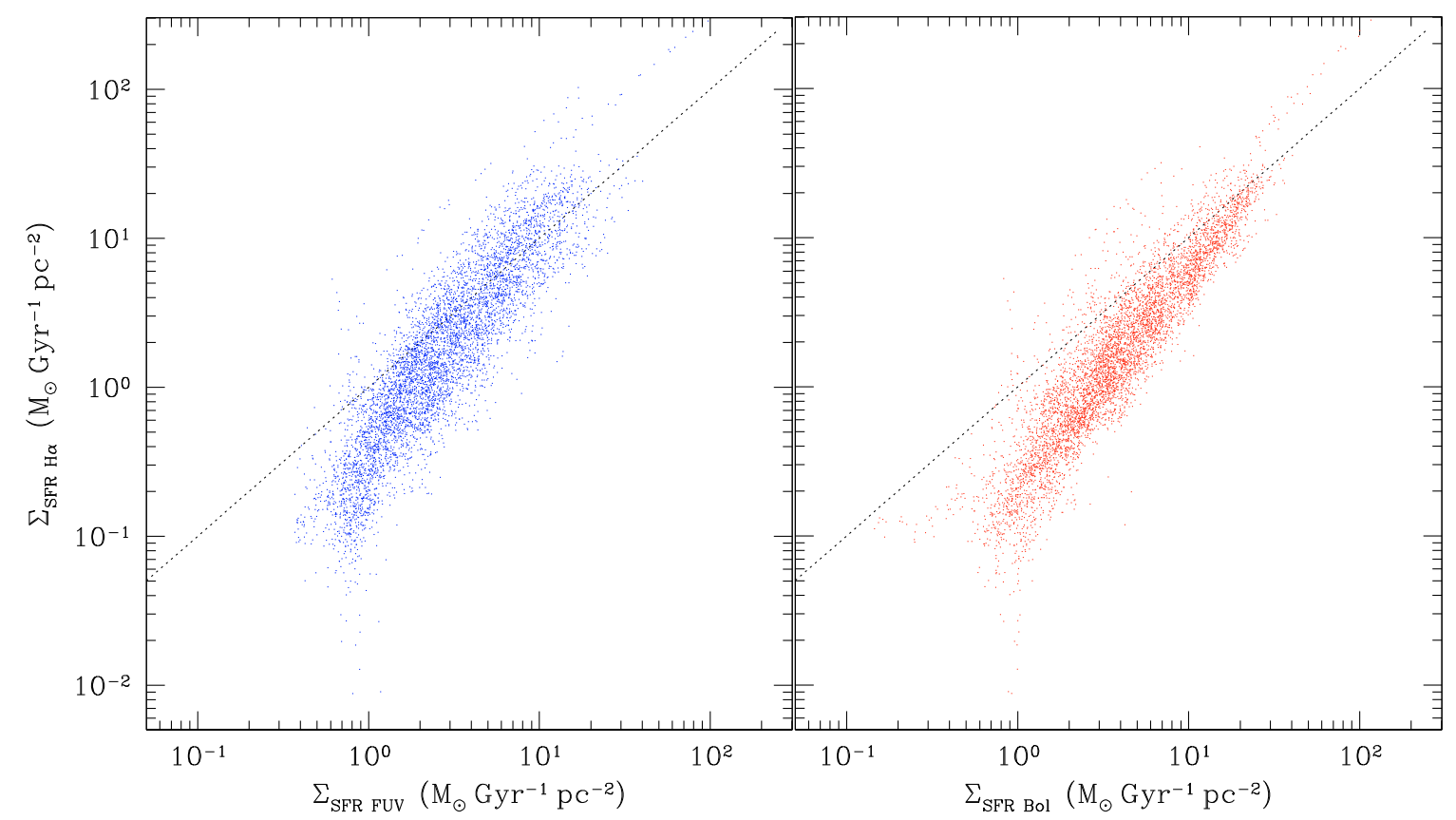

Fig. 8. $\Sigma_{\mathrm{SFR}}$ derived from $\mathrm{H} \alpha$ plotted against $\Sigma_{\mathrm{SFR}}(\mathrm{FUV})$ (left panel) and $\Sigma_{\mathrm{SFR}}$ (bol) (right panel). Only the 180 pc regions are shown. The dotted line corresponds to equality in the two SFRs.

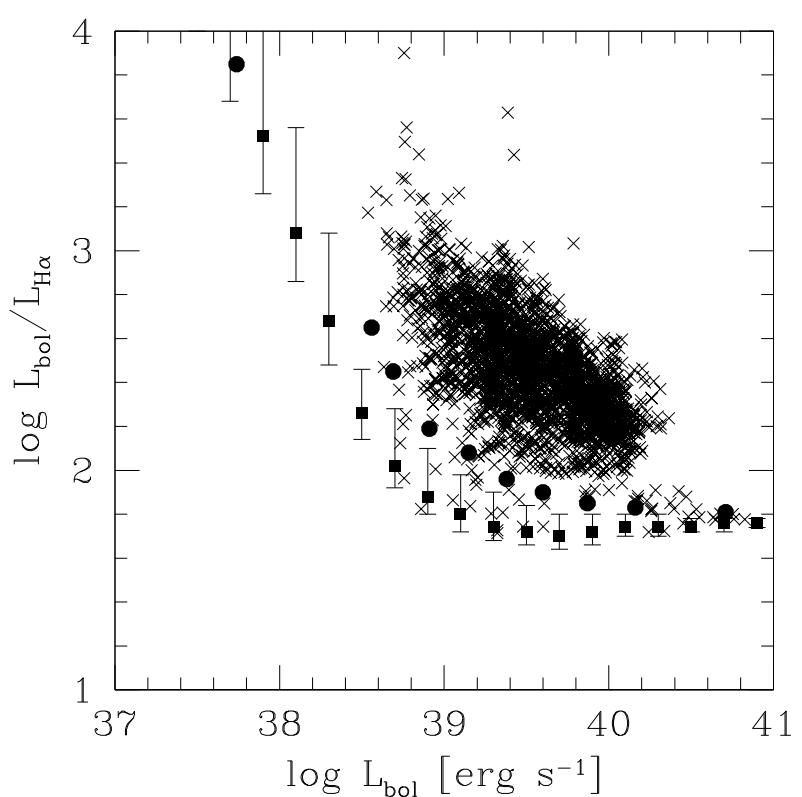

Fig. 9. The theoretical birthline (Paper III) which predicts a decreasing $\mathrm{H} \alpha$ flux for decreasing luminosity of young stellar clusters due to incompleteness of the IMF is shown with filled squares for the randomly sampled cluster model. The filled circles are the model prediction for clusters with a truncated IMF. Cross symbols refer to M 33 data, areas observed at $180 \mathrm{pc}$ resolution.

IMF. These data cannot be used to judge which of the two cluster models applies, because regions $180 \mathrm{pc}$ wide may contain more than one cluster and not necessarily young (see Corbelli et al. 2009 , to address this issue correctly).

The fast decrease of the $\mathrm{H} \alpha$ luminosity in regions of low luminosity, which do not contain massive clusters, implies that using a constant $\mathrm{H} \alpha$ luminosity - SFR conversion factor, the inferred SFR will be much lower than the effective one. If the inferred SFR in low density regions is lower, the index of the KS relation will be artificially higher. This explains the steeper indices we find when using $\mathrm{H} \alpha$ as the SFR indicator in the local study of M 33. We conclude that the lack of massive stars in low luminosity regions makes $\mathrm{H} \alpha$ an unreliable tracer of the SFR.

The birthline is the line where very young stellar clusters lie. Aging or leakage of ionizing photons bring the clusters above the birthline. Aging decreases $L_{\mathrm{H} \alpha}$ faster than $L_{\text {bol }}$ and moves $L_{\mathrm{bol}} / L_{\mathrm{H} \alpha}$ to higher values more or less vertically above the birthline (see Paper III). Also leakage of ionizing photons from $\mathrm{H}$ II regions raises the $L_{\mathrm{bol}} / L_{\mathrm{H} \alpha}$ values above the birthline. All our sampled regions (M 33 areas at 180 pc resolution) lie above the birthline (cross symbols) as they should, implying that we have not underestimated their bolometric luminosity. However, most of the SF regions we sample are well above the birthline, and this suggests that there is a mix of young and aged $\mathrm{H}$ II regions in each area and/or that there is leakage of ionizing photons. The aging and leakage processes and the approximate formula used to estimate $L_{\mathrm{bol}}$ and extinction correction to $L_{\mathrm{H} \alpha}$ explains the observed scatter in the $L_{\mathrm{bol}}-L_{\mathrm{H} \alpha}$ relation. M 33 is known to have a high diffuse fraction of $\mathrm{H} \alpha$ emission due to the leakage process from individual star-forming regions (Hoopes \& Walterbos 2000). But also in the continuum UV radiation M 33 has high diffuse fractions (Thilker et al. 2005), and if radiation escapes from the disk from these diffuse regions or from individual starforming sites, both non-ionizing and ionizing photons will contribute to this leakage. Hence although leakage can explain a possible steepening of the $\Sigma_{\mathrm{SFR}}$ (from all tracers) at low gas surface densities, it does not explain the non-linear scaling between $L_{\text {bol }}$ or $L_{\mathrm{FUV}}$ and $L_{\mathrm{H} \alpha}$.

\subsection{The correlation between the SFR and the dust optical depth}

We now use the dust opacity at $160 \mu \mathrm{m}, \tau_{160}$ derived as in Paper II, as an unbiased indicator of the gas column density. In M 33 the metallicity gradient is very shallow (Magrini et al. 2007b; Rosolowsky \& Simon 2008), and hence it is probable 


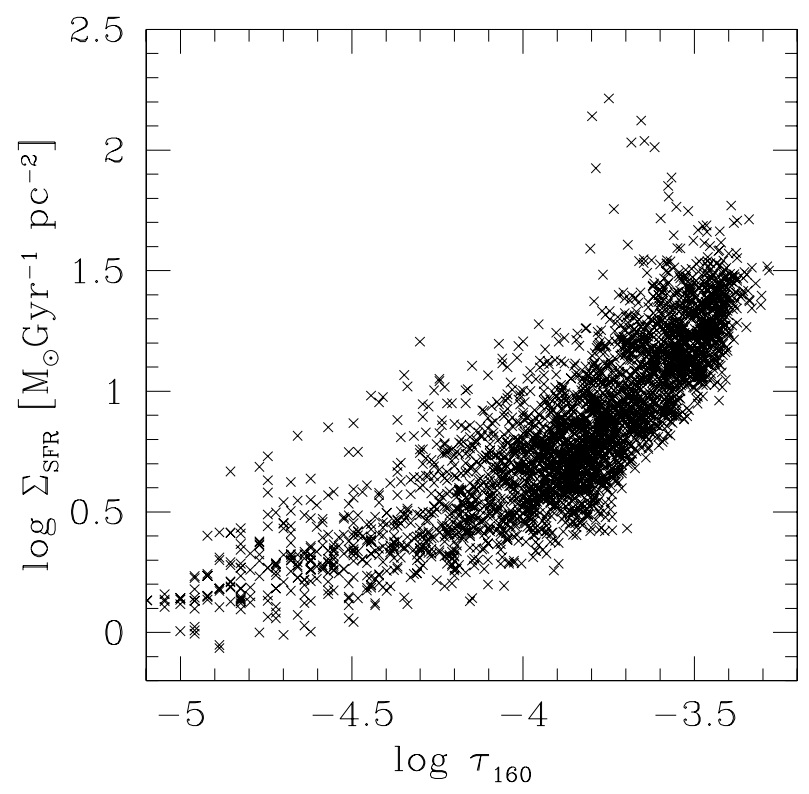

Fig. 10. Correlation between the SFR (FUV) surface density and the dust opacity at $160 \mu \mathrm{m}$. Cross symbols are data for areas in M 33 which have a $180 \mathrm{pc}$ wide diameter; only those points are shown with the CO luminosity above $2 \sigma$ detection threshold.

that the gas-to-dust ratio does not change much going radially outwards. The declining behavior of the dust-to-gas ratio found in Paper II when using the $21-\mathrm{cm}$ emission line brightness to infer the H I column density and the CO $J=1-0$ emission line brightness to infer the $\mathrm{H}_{2}$ column density, can then be due to an underestimation of the gas surface density at large galactocentric radii. In particular the $\mathrm{CO}-$ to- $\mathrm{H}_{2}$ conversion factor can be different from the value assumed in the M 33 FCRAO survey $\left(2.8 \times 10^{20} \mathrm{~cm}^{-2}\left(\mathrm{~K} \mathrm{~km} \mathrm{~s}^{-1}\right)^{-1}\right)$, and can vary across the M 33 disk according to local variations of the metal abundance and molecular clouds properties. Many papers in the literature (e.g. Leroy et al. 2007, 2009, and references therein) show that the $\mathrm{CO}-$ to- $\mathrm{H}_{2}$ conversion factor is effectively higher in low metallicity galaxies and can vary even in single molecular cloud complexes according to the cloud self-shielding conditions. As an alternative there could be opaque H I gas unaccounted for by the usual optically thin assumption for the $21-\mathrm{cm}$ line emission, as found in M 31 by Braun et al. (2009).

We show in Fig. 10 the correlation between the SFR surface density and the dust opacity. The Pearson linear correlation coefficient is much higher $(r=0.81)$ when considering the correlation in the $\log \Sigma_{\mathrm{SFR}}-\log \tau_{160}$ plane than when using the brightness of the $21-\mathrm{cm}$ and CO $J=1-0$ lines to derive $\log \Sigma_{\mathrm{SFR}}-\log \Sigma_{\mathrm{H}_{\mathrm{tot}}}$ relation $(r=0.43)$. As shown in Fig. 10 the distribution seems bimodal; there is an inflection in the slope around $\log \tau_{160} \approx-4$. The average slope (given in Table 3 ) and the correlation coefficient do not depend on whether we include positions where the CO luminosity is below the $2 \sigma$ limit. We have assumed a $20 \%$ error on $\tau_{160}$ to determine $n$, but given the high Pearson coefficient, the slope is not much dependent on the relative errors.

Although the correlation $\log \Sigma_{\mathrm{SFR}}-\log \tau_{160}$ is quite tight, some more work is needed to check the assumptions made and better address the implications of this result. For example even though the average radial variation of the metallicity is small $(\sim-0.4$ dex $)$, metallicity rises in the center (U et al. 2009), and at a given radius the variation in the oxygen abundance can be almost one order of magnitude ( - 0.8 dex) (Magrini et al. 2007a). Right now the relative errors on metal abundances are large, but if future measurements confirm this scatter, the assumption of a constant dust-to-gas ratio, implicit in the correlation, may not be appropriate. Also, the actual estimate of the optical depth is based on the intensity of the emission at $160 \mu \mathrm{m}$ and on the dust temperature derived using the 70 and $160 \mu \mathrm{m}$ emission. If the emission at $70 \mu \mathrm{m}$ is due to dust heated not only by the interstellar radiation field but also by radiation from star-forming regions, then we would expect a higher colour temperature. This would give a larger thermal intensity, and thus we would derive a smaller $\tau_{160}$, anti-correlated with the SFR. High-resolution IR observations at longer wavelengths, which will be available in the near future with Herschel, will allow a more accurate estimate of the dust optical depth. The resulting correlation coefficient involving $\log \tau_{160}$ and $\log \Sigma_{\text {SFR }}$ could have a higher value than the one derived here.

\section{Summary and conclusions}

We test the KS law in the Local Group spiral galaxy M 33, in azimuthally averaged areas (240 pc wide) and locally from $\sim 1.5 \mathrm{kpc}$ spatial resolution down to a resolution of $180 \mathrm{pc}$. Starting from a multiwavelength set of observations, we used the $\mathrm{H} \alpha, \mathrm{FUV}$, and bolometric (FUV+TIR) luminosities to estimate the SFR. For gas surface density we consider the molecular, atomic, and total gas phases. We use extinction-corrected SFR even though extinction marginally affects the observed properties of the optical or ultraviolet emissions in M 33 because of the rather low dust content of the galaxy. The most important results are summarized below:

- At every spatial scale we find that the $\mathrm{H} \alpha \mathrm{KS}$ indices are always higher than the FUV and bolometric ones, and we explain this as due to the lack of $\mathrm{H} \alpha$ emission in low luminosity regions where most of stars form in small clusters with an incomplete initial mass function at their high mass end. We use the cluster birthline to support this, which implies a non-linear relation between the $\mathrm{H} \alpha$ and bolometric luminosities. The birthline also shows that most regions, even at the highest spatial resolution, contain a mixture of ages which on average are smaller than $10 \mathrm{Myr}$.

- For azimuthally averaged values, the depletion timescale for the molecular gas is radially constant and the KS index is $1.1 \pm 0.1$ for both FUV and bolometric SFR tracer, lower than that found by Heyer et al. (2004) using IR SFR tracers alone. The depletion time for the molecular gas is relatively constant, with a value of about 1 Gyr. The correlations with the molecular and total gas are tighter than with the H I gas, despite the fact that most of the gaseous mass of M 33 is in H I form. Denser filaments of neutral gas are found where star formation takes place, but their H I surface density does not correlate with the SFR. The KS radial index for the total gas surface density is $2.9 \pm 0.2$. The depletion time for the total gas density widely varies from $\sim 0.5 \mathrm{Gyr}$ in the center of the galaxy to about $10 \mathrm{Gyr}$ in the outer parts.

- Locally the dispersion in the SFR-gas density relation per unit surface is high, and results are very sensitive to the statistical method used to fit the data. The scatter in the KS relation increases as the spatial resolution increases. At $180 \mathrm{pc}$ resolution, fitting the $\log \Sigma_{\mathrm{SFR}}-\log \Sigma_{\mathrm{H}_{2}}$ data to a straight line using a recursive fitting method which removes outlier points, we obtain the same the KS index than in the radial 
average analysis (1.1). There is a slight general tendency for the local KS laws to show higher slopes for coarser spatial resolutions. As for radial averages, the local KS index for the molecular gas is much lower than that for the total gas.

- For our finest resolution (180 pc) we also derive the best fitting straight line to the data in the $\log -\log$ plane, taking into account the errors in the determination of the gas surface density as well as the errors in the determination of the SFR as traced by FUV emission. Considering only the positions where the $\mathrm{CO}$ detection is above $2 \sigma$ noise, a bivariate regression gives the KS index for the molecular gas of $2.22 \pm$ 0.07 with a Pearson linear correlation coefficient of 0.42 . We believe that given the large scatter in the $\log \Sigma_{\mathrm{SFR}}-\log \Sigma_{\mathrm{H}_{2}}$ relation at the highest spatial resolution examined in this paper, this method gives more robust result. This KS index is higher than the one we find using the recursive fit and all positions in our maps. It is also higher than what the ordinary-leastsquare method gives for the same set of data (no uncertainties considered). The bivariate regression gives a slope for the $\log \Sigma_{\mathrm{SFR}}-\log \Sigma_{\mathrm{H}_{\mathrm{tot}}}$ relation only slightly higher than for the molecular gas alone: $2.64 \pm 0.07$.

- Given the rather poor correlations between the gas and the SFR per unit surface, we analyze whether the SFR correlates with other physical quantities. A good correlation is found with the hydrostatic pressure, i.e. with the interstellar medium volume density considering a constant sound speed. This implies that the stellar disk, gravitationally dominant with respect to the gaseous disk in M 33, plays a major role in driving the SFR. The slope of the correlation is close to unity, suggesting that the SFR per unit area (or per unit volume if the thickness of the SF disk does not vary) is proportional to the ISM volume gas density. Since the correlation is tighter, its slope is less dependent on the statistical method used.

- There is a good correlation between the dust optical depth at $160 \mu \mathrm{m}$ and the local SFR density. This can be interpreted in terms of a $\Sigma_{\mathrm{SFR}}-\Sigma_{\text {gas }}$ relation if the dust opacity is used as an unbiased indicator of the surface density of gas prone to star formation. This might be the case for example if the gas-to-dust ratio were constant in M 33 while the $\mathrm{CO}-$ to- $\mathrm{H}_{2}$ conversion factor were not, or if there were opaque $\mathrm{HI}$ gas unaccounted for by the usual optically thin assumption for the $21-\mathrm{cm}$ line emission. The tightness of the $\log \Sigma_{\mathrm{SFR}}-\log \tau_{160}$ correlation and the shallower slope compared to the $\log \Sigma_{\text {SFR }}-\log \Sigma_{\text {gas }}$ relation would then imply that the KS law in low luminosity galaxies still holds, but the surface density of the gas in the process of forming stars has a wider dynamical range than shown by the intensity of the $21-\mathrm{cm}$ and CO $J=1-0$ lines. Still, some caution is called for in interpreting this result because of some of the assumptions made in deriving it. A more detailed analysis is required together with a more accurate determination of the dust optical depth, which will be possible in the near future thanks for example to the Herschel satellite data.

Acknowledgements. We would like to thank Rene Walterbos for providing us the $\mathrm{H} \alpha$ image of M 33, R. Kennicutt and G. Helou for interesting discussions, and the anonymous referee for comments that helped to improve our work. The work of S. V. was supported by a INAF - Osservatorio Astrofisico di Arcetri fellowship. The Spitzer Space Telescope is operated by the Jet Propulsion Laboratory, California Institute of Technology, under contract with the National Aeronautics and Space Administration. This research has made use of the NASA/IPAC Extragalactic Database, which is operated by JPL/Caltech, under contract with NASA.

\section{References}

Bell, E. F. 2003, ApJ, 586, 794

Bigiel, F., Leroy, A., Walter, F., et al. 2008, AJ, 136, 2846

Blitz, L., \& Rosolowsky, E. 2005, in The Initial Mass Function 50 Years Later, ed. E. Corbelli, F. Palla, \& H. Zinnecker, Astrophys. Space Sci. Libr., 327, 287

Blitz, L., \& Rosolowsky, E. 2006, ApJ, 650, 933

Boissier, S., Prantzos, N., Boselli, A., \& Gavazzi, G. 2003, MNRAS, 346, 1215 Boissier, S., Gil de Paz, A., Boselli, A., et al. 2007, ApJS, 173, 524

Braun, R., Thilker, D. A., Walterbos, R. A. M., \& Corbelli, E. 2009, ApJ, 695, 937

Calzetti, D. 2001, PASP, 113, 1449

Calzetti, D., Kennicutt, Jr., R. C., Bianchi, L., et al. 2005, ApJ, 633, 871

Corbelli, E. 2003, MNRAS, 342, 199

Corbelli, E., \& Schneider, S. E. 1997, ApJ, 479, 244

Corbelli, E., \& Walterbos, R. A. M. 2007, ApJ, 669, 315

Corbelli, E., Verley, S., Elmegreen, B. G., \& Giovanardi, C. 2009, A\&A, 495, 479

Dale, D. A., \& Helou, G. 2002, ApJ, 576, 159

Deul, E. R., \& van der Hulst, J. M. 1987, A\&AS, 67, 509

Elmegreen, B. G. 1993a, ApJ, 419, L29

Elmegreen, B. G. 1993b, ApJ, 411, 170

Engargiola, G., Plambeck, R. L., Rosolowsky, E., \& Blitz, L. 2003, ApJS, 149, 343

Gardan, E., Braine, J., Schuster, K. F., Brouillet, N., \& Sievers, A. 2007, A\&A, 473, 91

Gil de Paz, A., Boissier, S., Madore, B. F., et al. 2007, ApJS, 173, 185

Greenawalt, B. E. 1998, Ph.D. Thesis, AA, New Mexico State University

Guibert, J., Lequeux, J., \& Viallefond, F. 1978, A\&A, 68, 1

Heyer, M. H., Corbelli, E., Schneider, S. E., \& Young, J. S. 2004, ApJ, 602, 723

Hoopes, C. G., \& Walterbos, R. A. M. 2000, ApJ, 541, 597

Iglesias-Páramo, J., Buat, V., Takeuchi, T. T., et al. 2006, ApJS, 164, 38

Isobe, T., Feigelson, E. D., Akritas, M. G., \& Babu, G. J. 1990, ApJ, 364, 104

Kennicutt, Jr., R. C. 1989, ApJ, 344, 685

Kennicutt, Jr., R. C. 1998a, ARA\&A, 36, 189

Kennicutt, Jr., R. C. 1998b, ApJ, 498, 541

Kennicutt, Jr., R. C., Calzetti, D., Walter, F., et al. 2007, ApJ, 671, 333

Koyama, H., \& Ostriker, E. C. 2009, ApJ, 693, 1316

Krumholz, M. R., McKee, C. F., \& Tumlinson, J. 2009, ApJ, 699, 850

Leitherer, C., Schaerer, D., Goldader, J. D., et al. 1999, ApJS, 123, 3

Leroy, A., Bolatto, A., Stanimirovic, S., et al. 2007, ApJ, 658, 1027

Leroy, A. K., Bolatto, A., Bot, C., et al. 2009, ApJ, 702, 352

Madore, B. F., van den Bergh, S., \& Rogstad, D. H. 1974, ApJ, 191, 317

Magrini, L., Corbelli, E., \& Galli, D. 2007a, A\&A, 470, 843

Magrini, L., Vílchez, J. M., Mampaso, A., Corradi, R. L. M., \& Leisy, P. 2007b, A\&A, 470, 865

Makovoz, D., \& Marleau, F. R. 2005, PASP, 117, 1113

Martin, C. L., \& Kennicutt, Jr., R. C. 2001, ApJ, 555, 301

Martin, D. C., Fanson, J., Schiminovich, D., et al. 2005, ApJ, 619, L1

McConnachie, A. W., Chapman, S. C., Ibata, R. A., et al. 2006, ApJ, 647, L25

McKee, C. F., \& Tan, J. C. 2003, ApJ, 585, 850

Newton, K. 1980, MNRAS, 190, 689

Press, W. H., Teukolsky, S. A., Vetterling, W. T., \& Flannery, B. P. 1992, Numerical recipes in FORTRAN, The art of scientific computing, ed. W. H. Press, S. A. Teukolsky, W. T. Vetterling, \& B. P. Flannery

Rieke, G. H., Young, E. T., Engelbracht, C. W., et al. 2004, ApJS, 154, 25

Robertson, B., \& Kravtsov, A. 2008, ApJ, 680, 1083

Rosolowsky, E., \& Simon, J. D. 2008, ApJ, 675, 1213

Rosolowsky, E., Engargiola, G., Plambeck, R., \& Blitz, L. 2003, ApJ, 599, 258

Rosolowsky, E., Keto, E., Matsushita, S., \& Willner, S. P. 2007, ApJ, 661, 830

Schmidt, M. 1959, ApJ, 129, 243

Schmidt, M. 1963, ApJ, 137, 758

Thilker, D. A., Hoopes, C. G., Bianchi, L., et al. 2005, ApJ, 619, L67

Thilker, D. A., Boissier, S., Bianchi, L., et al. 2007, ApJS, 173, 572

Toomre, A. 1964, ApJ, 139, 1217

U, V., Urbaneja, M. A., Kudritzki, R., et al. 2009, ApJ, 704, 1120

Verley, S., Hunt, L. K., Corbelli, E., \& Giovanardi, C. 2007, A\&A, 476, 1161

Verley, S., Corbelli, E., Giovanardi, C., \& Hunt, L. K. 2009, A\&A, 493, 453

Walter, F., Brinks, E., de Blok, W. J. G., et al. 2008, AJ, 136, 2563

Ward-Thompson, D., Scott, P. F., Hills, R. E., \& Andre, P. 1994, MNRAS, 268, 276

Werner, M. W., Roellig, T. L., Low, F. J., et al. 2004, ApJS, 154, 1

Wong, T., \& Blitz, L. 2002, ApJ, 569, 157

Wright, M. C. H., Warner, P. J., \& Baldwin, J. E. 1972, MNRAS, 155, 337 